\title{
Potential therapeutic approaches for modulating expression and accumulation of defective lamin $A$ in laminopathies and age-related diseases
}

\author{
Alex Zhavoronkov • Zeljka Smit-McBride • \\ Kieran J. Guinan • Maria Litovchenko • \\ Alexey Moskalev
}

Received: 6 May 2012 /Revised: 8 September 2012 / Accepted: 25 September 2012 / Published online: 23 October 2012

(C) The Author(s) 2012. This article is published with open access at Springerlink.com

\begin{abstract}
Scientific understanding of the genetic components of aging has increased in recent years, with several genes being identified as playing roles in the aging process and, potentially, longevity. In particular, genes encoding components of the nuclear lamina in eukaryotes have been increasingly well characterized, owing in part to their clinical significance in age-related diseases. This review focuses on one such gene, which encodes lamin A, a key component of the nuclear lamina. Genetic variation in this gene can give
\end{abstract}

A. Zhavoronkov $(\bowtie) \cdot$ M. Litovchenko

Bioinformatics and Medical Information Technology Laboratory,

Center for Pediatric Hematology, Oncology and Immunology,

Moscow 119296, Russia

e-mail: alex@biogerontology.org

A. Zhavoronkov $\cdot$ K. J. Guinan · A. Moskalev

The Biogerontology Research Foundation,

Reading, UK

e-mail: guinank@tcd.ie

e-mail: amoskalev@list.ru

\section{Z. Smit-McBride}

Department of Ophthalmology and Vision Science,

School of Medicine, University of California at Davis,

Davis, CA 95616, USA

e-mail: zsmcbride@ucdavis.edu

\section{K. J. Guinan}

BioAtlantis Ltd., Kerry Technology Park,

Tralee, County Kerry, Ireland

\section{A. Moskalev}

Laboratory of Molecular Radiobiology and Gerontology,

Institute of Biology, Komi Science Center of Russian

Academy of Sciences,

Syktyvkar 167982, Russia rise to lethal, early-onset diseases known as laminopathies. Here, we analyze the literature and conduct computational analyses of lamin A signaling and intracellular interactions in order to examine potential mechanisms for altering or slowing down aberrant Lamin $A$ expression and/or for restoring the ratio of normal to aberrant lamin A. The ultimate goal of such studies is to ameliorate or combat laminopathies and related diseases of aging, and we provide a discussion of current approaches in this review.

Keywords Lamin A · Progeria · Laminopathies ·

Age-related diseases $\cdot$ Aging

\section{Introduction}

The nuclear lamina is an array of intermediate filament proteins inside the nucleus of eukaryotic cells, which supports the structure of the nucleus, including its shape and mechanical stability $[1,2]$. In addition, it serves as a scaffold for the attachment of DNA-protein complexes that regulate both eu- and heterochromatin histone modifications [3]. The nuclear lamina is involved in the regulation of many key biological processes, including DNA replication, transcription, cell cycle progression, and chromatin organization [2]. Given the central role of the nuclear lamina in such a wide range of essential processes, it is not surprising that alterations in the structure can have a significant impact on normal cellular function, and in some cases can give rise to disease and even mortality within affected organisms.

Maintenance of the nuclear lamina is essential for most eukaryotic life forms, and requires the presence of an array 
of specific proteins that are highly conserved evolutionarily, both in terms of their structure and function. In particular, major functional components of the nuclear lamina are fibrous proteins known as nuclear lamins, which support this structure through interactions with specific membrane-associated proteins. Lamins are highly conserved evolutionarily, being represented in all examined metazoan life forms; thus, their essential functions likely ensure survival across a broad range of species [4]. Mutations within lamin genes and subsequent alterations in the structure and function of the proteins they encode can give rise to a broad range of diseases known as laminopathies. Such diseases are characterized by a broad range of severe clinical symptoms and complications, with some causing mortality early in life. Numerous laminopathies have been identified in humans during the last decade, and have been linked to several types of mutations in causative loci, both within lamin genes themselves and in genes encoding lamin-binding proteins. Laminopathies include Emery-Dreifuss muscular dystrophy (MIM 181350), dilated cardiomyopathy (MIM 115200), familial partial lipodystrophy (MIM 151660), Charcot-Marie-Tooth disorder type 2B1 (MIM 605588), Greenberg skeletal dysplasia (MIM 215140), limb girdle muscular dystrophy Type 1B (MIM 159001) and mandibuloacral dysplasia with type A lipodystrophy (MIM 248370). The molecular mechanisms by which lamins contribute to these diseases have become increasingly understood in recent years, particularly in terms of the genetic mutations and effects therein on both gene expression and protein structure and function.

\section{Lamin A processing, mutations, and role in diseases}

Lamins can be categorized as either A type (lamins A and C) or B type (lamins B1 and B2). In humans, A-type lamins are encoded by a single gene-LMNA (Entrez Gene ID: 4000)-located on chromosome 1q21.2, while B-type lamins are encoded by two genes- LMNB1 and LMNB2 (Entrez Gene ID: 4001 and 84823)-located on chromosomes 5q23.2 and 19p13.3, respectively. The processes involved in the expression of lamin genes and their translation and processing into mature and functional proteins include a series of specific and essential steps, alterations to which can impact the essential molecular and cellular functions of these proteins. In the case of LMNA, one essential step in protein biosynthesis and maturation is farnesylation at the $\mathrm{C}$-terminus by the enzyme farnesyltransferase [5]. This posttranslational modification plays a role in targeting prelamin $\mathrm{A}$ to the inner nuclear membrane. Farnesylation is followed by several steps involving the endoproteolytic cleavage of the last three amino acids by zinc metallopeptidase ZMPSTE24, carboxymethylation of the C-terminal cysteine by ICMT methyltransferase, and proteolytic removal of the last 18 amino acids by ZMPSTE24, resulting in the removal of the farnesyl tail on the $\mathrm{C}$-terminus [6, 7]. Mature lamin A is then released from its membrane anchor, which allows it to be properly positioned in the nuclear scaffold. Factors that interfere with these steps in such a way as to affect lamin maturation can have negative effects on nuclear lamin and can ultimately lead to an array of downstream effects, detrimental to cellular health and in some cases, longevity.

Within the LMNA gene alone, over 400 different point mutations have been identified, many of which are underlying causes of laminopathies [8,9], including restrictive dermopathy (MIM 275210) and Hutchinson-Gilford progeria syndrome (HGPS; MIM 176670). HGPS presents as a broad range of clinical features, which most notably include accelerated aging [10]. HGPS is caused by mutations in the LMNA gene, the most well-known of which is a de novo heterozygous point mutation in position $1824 \mathrm{C}>\mathrm{T}$ (G608G) [11]. While the $G 608 G$ mutation does not cause any change in the encoded amino acid, it does activate a cryptic splice donor site in exon 11 of the LMNA gene. Consequently, a splice variant of Prelamin A mRNA is generated with an internal deletion of 150 base pairs [11]. These transcripts are translated into progerin, the truncated form of the lamin A protein, with a 50 amino acid internal deletion near the C-terminus [11]. The internal deletion eliminates the essential endoprotease ZMPSTE24 recognition site, resulting in progerin remaining permanently farnesylated and anchored to the nuclear membrane $[11,12]$. The accumulation of progerin in cells of patients carrying the G608G mutation severely impacts the structure of the nuclear lamina, culminating in the cellular and disease phenotypes characteristic of HGPS.

Severe forms of progeria also occur due to a number of other mutations in $L M N A$, such as $1821 \mathrm{G}>\mathrm{A}$ and $1,968 \mathrm{G}$ $>\mathrm{A}$, mutations associated with increased ratios of progerin to normal, wild-type protein [13]. An extremely severe case of neonatal progeria in which death occurs within the first year of life has recently been found to be associated with heterozygosity $(1,821 \mathrm{G}>\mathrm{A})$. Examination of patient fibroblasts demonstrates an increased ratio of progerin to lamin A, relative to those levels typically observed in HGPS, suggesting that disease severity may be determined in part by the ratio of the farnesylated protein to mature lamin A $[13,14]$.

The hallmarks of progeria and its characteristic phenotypes are broadly associated with alterations in the production of progerin relative to mature lamin A, imbalances that directly impact key biological processes occurring at both the genetic and cellular levels. Progerin is observed to 
accumulate in all tissues of HGPS patients, acting as a dominant-negative protein that significantly modifies the structure of the nuclear lamina [15]. The cellular phenotype of HGPS patients includes nuclear blebbing, thinning of the nuclear lamina, loss of peripheral heterochromatin, and clustering of nuclear pores [16]. Accumulation of progerin in HGPS as nucleoplasmic aggregates leads to inhibition of the transport of several factors that play key roles in the functioning of the nucleus [15]. Examination of fibroblast cells from patients with HGPS demonstrates deficiencies in histone modification, alterations in gene expression, delays in the response to DNA damage, disturbances of mitosis, and cytokinesis, abnormalities in chromosome segregation and increases in the occurrence of binucleated cells [9]. The hallmarks and clinical features of HGPS are therefore deeply rooted in alterations taking place at genetic and protein biosynthesis levels and, in turn, those subsequent changes that negatively impact key biological processes further downstream. While there are significant components to HGPS that are associated with aging, disease pathogenesis, and progression is likely to involve several factors not exclusive to the aging process. While many tissues in HGPS patients exhibit phenotypes associated with accelerated aging, not all tissues are typically affected (reviewed by [17]). In addition, HGPS may not be viewed exclusively as a disease of accelerated aging, given that certain aspects of the disease are not typically associated with normal aging; for example, the presence of clavicular agenesis. Significant features of HGPS that are associated with normal aging include increases in DNA damage, defects in DNA repair, alterations in telomeric dynamics, and increases in cell proliferation, senescence, and tissue homeostasis (reviewed by [17] and references therein). In this respect, HGPS may be viewed as a disease that substantially resembles premature aging, but does not include all aspects of it, and is segmental in nature.

\section{The significance of LMNA in human health and longevity}

Several genes have been identified in recent years as influencing the aging process and possibly longevity $[18,19]$. The potential significance of the $L M N A$ gene in human health and its potential contribution to susceptibility to many common diseases is also becoming increasingly appreciated. In particular, Scaffidi et al. demonstrate that the molecular mechanism that underlies HGPS also takes place in normal cells at a lower rate [20]. The nuclei of cells of normal-aged individuals exhibit defects similar to those of cells of HGPS patients, including changes in histone modification and increased levels of DNA damage. Age-dependent defects in the nuclei of cells of healthy individuals are caused by infrequent use of the same cryptic splice site of Lamin A, whose constitutive activation generates a Progerin transcript [20]. The overexpression of normal Prelamin A can lead to growth defects in human vascular smooth muscle cells [21], similar to those changes observed in cells producing Progerin [22]. Cytotoxicity can also be induced by a minor increase in the steadystate level of one or more intermediate products of Prelamin A processing [12, 22].

Recent years have shown extensive investigation of the potential contribution of genetic variability within lamin genes to disease susceptibility. Disease-association studies including SNPs at lamin loci, have implicated metabolic syndrome, dislipidemia, type-II diabetes, obesity, polycystic ovary syndrome, arterial stiffness, and vascular disease [23-35]. In addition, there is some evidence for the potential influence of genetic variation at $L M N A$ on human longevity and age-related diseases [36-38]. Findings from these studies have been variable, with the majority focusing on the 1908C > T; rs4641 LMNA SNP. rs4641 has been found in several cases to be significantly associated with disease susceptibility and related conditions across a number of ethnically diverse population cohorts for type II diabetes and related diseases [23, 24, 26-28, 31]. The rs4641 SNP is a silent $\mathrm{C}>\mathrm{T}$ substitution occurring at exon 10 of the $L M N A$ gene, the exon in which alternative splicing gives rise to mRNAs that code for either Prelamin A or Lamin C [39]. The mechanism by which this SNP alters the LMNA gene product and phenotype to potentially influence susceptibility to these diseases is unknown. However, recent evidence suggests that the $\mathrm{C}$ and $\mathrm{T}$ alleles of rs4641 are associated with differential gene expression phenotypes, with the $\mathrm{C}$ allele associated with increased levels of transcripts of Lamin A and Lamin C relative to those detected for the $\mathrm{T}$ allele [40]. While this study demonstrates that differential, allele-specific expression is present at the LMNA locus in HGPS, it is unclear whether or not such variability is directly associated with the rs4641 SNP or if it is rather associated with other variants located within the same haplotype block. In light of these studies, the relevance of genetic variation at the $L M N A$ locus to more common diseases affecting populations at large may be significant. Interestingly, the rs4641 SNP is represented in all populations that have been examined in the HapMap project to date, with the minor ' $\mathrm{T}$ ' allele represented at levels ranging from between 5 and $10 \%$ in African populations, 20-25\% in Europeans and 23-32 \% in South and East Asian populations (www.hapmap.org) [41]. Elucidating the role of this relatively common SNP in disease pathogenesis, longevity, or related diseases, therefore, may have broad significance. However, in-depth examination of linkage disequilibrium between rs4641 and other functional SNPs is required to delineate the role of this LMNA SNP in human diseases, metabolic-related, age-related, or otherwise. 
Given the evidence that genetic variation at LMNA contributes to both laminopathies and more common human diseases, the identification of methods that can therapeutically alter $L M N A$ structure and restore a healthy homeostatic balance of aberrant/normal LMNA, warrants further investigation. A multifaceted approach is required to increase knowledge in this area and further elucidate the functional relevance and complex characteristics of lamins both in terms of their expression and functional interactions. In this way, interventions may be designed and developed to intervene, treat, and ameliorate symptoms of human diseases, particularly those associated with aging.

\section{Targeting LMNA and associated diseases: a multifaceted approach}

Combating the broad range of consequences associated with missplicing lamins and altering their gene expression levels requires a multifaceted approach, which includes targeting components at the genetic level as well as targeting components downstream cell signaling and cellular-level processes. Furthermore, unraveling the underlying complexity at each of these levels and, in turn, targeting specific processes therein to treat or ameliorate disease symptoms, requires (a) an in-depth knowledge of the molecular interactions between nuclear lamins and other proteins and cellular events, (b) in vitro and in vivo studies demonstrating effectiveness of the treatment and validating such interventions, and (c) development and refinement of techniques to manage, limit, and potentially reverse damage that has already been incurred in patients. Here, we perform an analysis of the existing literature and published data sets, with the goal of identifying novel targets for treating laminopathies and associated diseases. In turn, we hope that these findings may provide a basis for future experimental design, interpretation of results, and refinement of methods aimed at tackling severe laminopathies and other age-related diseases.

\section{Computational analysis of LMNA signaling and intracellular interactions}

In this study, we utilized literature searches of the NIH's PubMed database in order to examine pathways that regulate $L M N A$ expression. This was accomplished by using the following keywords during searches: gene expression regulation Lamin A/C or Progerin, and progeria. In order to visualize the molecular interactions between nuclear lamins and other proteins and identify novel targets, the Ingenuity Pathway Analysis (IPA, Ingenuity ${ }^{C}$ Systems, www.ingenuity.com) software and knowledgebase and its pathway designer graphical module were utilized. IPA provided graphical representations of network interactions of the LMNA protein with molecules involved in signal transduction pathways and other intracellular regulatory networks. Data used to generate pathways and interaction networks in IPA are compiled from interactions validated in multiple model organisms from peerreviewed journals by a team of IPA scientists. Advantages of this software tool include: (a) each connection displayed on a graph is documented by a peer-reviewed article, which can be examined by clicking on the relevant connection and (b) the Pathway Designer module contains "cell art" elements which can be used to graphically display connection locations (nucleus, mitochondria, cellular membrane, etc.).

Searches did not identify signaling or metabolic pathways in IPA that center on LMNA. However, LMNA was found to be part of a canonical Apoptosis Signaling pathway as a target of caspase 6 . The LMNA Interactome generated through this analysis displays 110 direct molecular interactions with LMNA, with all the known molecules that LMNA/lamin A interacts with, including proteins, protein modifiers, small molecules, and microRNAs. Some of these molecules are members of other signal transduction pathways, and therefore represent a bridge between LMNA and these pathways. The most important signal transduction pathways that target/affect LMNA are shown in Fig. 1. Data shown in this figure was generated by combining information from several individual canonical pathways, the LMNA Interactome, and additional information from the published literature, and then repeating several iterations of this process to reach a final model. As shown in Fig. 1, WNT/betacatenin, TGF beta, Notch, and PI3K represent the key signaling pathways upstream of $L M N A$, which likely regulate its expression. The main molecules that interact with the lamin A protein, and have genetic correlates with some of the laminopathies we have discussed here, are mainly found in the nucleus, including Sun1 and Sun2, whose potential roles in HGPS disorder have been investigated recently [42]. Many of the identified signaling pathways and molecules that interact with lamin A are known to exert effects on nuclear lamins by altering their expression levels. To visualize gene expression regulatory points that potentially may be targeted for intervention, the Pathway Designer module was applied to the data presented in Fig. 1. Potential interventions might include following mechanisms: transcription, splicing, translation, posttranslational modification, and degradation via autophagy. Using the overlay function of IPA, this figure was overlaid with a number of pharmaceuticals and drugs that may be used to target key proteins of any given biological process. While a number of drugs were identified via the IPA database, more detailed lists of drugs and agents that may be applicable are listed in Tables 1, 2, 3, 4 and 5 generated through PubMed searches using relevant keywords. To expand the literature search and identify additional known drugs and experimental 
compounds, as well as their side effects, potentially acting on elements of the pathways involving LMNA gene, we employed a manually curated proprietary database (MetaCore $^{\mathrm{TM}}$, GeneGo), and the MetaCore pathway analysis software.

\section{Targeting Lamin $A$ and Progerin expression via signal transduction pathways}

On the basis of the computational analysis outlined above, a number of potential targets and therapeutic interventions have been identified and discussed. Many of these potential interventions may nonspecifically down-regulate the level of expression of both Lamin A and its disease-associated allelic variants, while others have more specific effects on mutant Lamin A expression.

\section{Restoration of IGF-1 and GH balance}

Insulin-like growth factor 1 (IGF-1) signaling is involved in aging and longevity in many animals, including nematodes, Drosophila, and mammals (for review see [43]). Zmpste24 $(-/-)$ mice, a mouse model of progeria, exhibits dysregulation of somatotropic axis, characterized by high levels of circulating growth hormone (GH) and reductions in insulinlike growth factor-1 (IGF-1) [44]. Application of recombinant IGF-1 restores the balance between IGF-1 and GH, and this delays the onset of several progeroid characters and prolongs the lifespan of progeroid animals [44, 45]. However, applying such an approach as a means of treating HGPS may be limited, given the diverse biological effects exerted by this hormone, and in particular, the pathogenic role of IGF-1 signaling in cancer [46].

\section{Notch signaling inhibitors}

Expression of Progerin ectopically activates effectors of Notch and downregulates the canonical Wnt signaling pathway, regulating the differentiation of mesenchymal stem cells $[47,48]$. This leads to misregulation of somatic stem cell differentiation, explaining some of the pathological defects of HGPS [49]. Thus, inhibitors of Notch signaling (Table 1) and recombinant $\beta$-catenin could potentially ameliorate symptoms of HGPS. Notch 2 may also be targeted for inhibition given its influence on Granzyme B transcription [50] and potentially apoptosis; however, the impact of such an approach on immune function is unclear. Furthermore, there are severe side effects known to be associated with certain Notch inhibitors, including gastrointestinal bleeding and skin cancer [51]. Therefore, caution must be taken when applying such approaches to treating HGPS patients.
Reactive oxygen species scavengers

Basal levels of reactive oxygen species (ROS) as well as induced levels of $\mathrm{H}_{2} \mathrm{O}_{2}$ are five times higher in HGPS fibroblasts compared to normal fibroblasts, which leads to double stranded breaks (DSBs) in DNA and a decrease in the proliferative capacity of cells [52]. Indeed, HGPS is accompanied by an elevated quantity of DSBs and attenuation of their repair [53]. On the contrary, the ROS scavenger $\mathrm{N}$-acetyl cysteine (NAC) has been shown to decrease basal levels of DSBs and enhance population-doubling times in fibroblasts derived from HGPS patients [52]. Other effective ROS scavengers are listed in Table 2. However, the effectiveness of using ROS scavengers for treating progeria patients and ameliorating intracellular damage and associated symptoms remains somewhat speculative. In addition, the complex biochemical effects of some anti-oxidants may raise some safety concerns. While the anti-oxidative effects of ascorbic acid are well characterized, pro-oxidative effects have also been described [54-56] and such properties must be taken into consideration when developing treatments for progeria.

\section{Telomerase activators}

Progressive attrition of telomeres causes activation of progerin production in normal human fibroblasts [9]. Active telomerase prolongs the cellular lifespan of HGPS by decreasing progerin-induced DNA-damage signaling and activation of both the $\mathrm{p} 53$ and $\mathrm{Rb}$ pathways. These are the two pathways that mediate the onset of premature senescence in HGPS [57]. Telomerases can be stimulated by a potent telomerase activator, TA-65, to extend short telomeres, and this has been shown to have a positive effect on health span in mice [58]. Treatment with TA-65 may prevent some symptoms of HGPS. However, while the results in mice are promising, the use of this technology in humans may be limited, particularly in the event of any significant side effects being identified in future clinical trials.

\section{$\mathrm{Rb}$ regulators}

Progerin accumulation leads to premature replicative cellular senescence [59]. Marji et al. suggest, based on global gene profiling of HGPS fibroblasts, that defects in the lamin $\mathrm{A}-\mathrm{Rb}$ signaling pathway may be key factors in the accelerated aging phenotype of HGPS, and perhaps in normal aging, too [60]. Rb activity can be modified with reagents such as roscovitine and PD-0332991, inhibitors of Cdk2cyclin E and Cdk4/cyclin D1 complexes, respectively, that phosphorylate and inactivate the $\mathrm{Rb}$ tumor suppressor [61]. However, studies indicate that $\mathrm{Rb}$ expression is decreased in fibroblasts in both HGPS and normal aging, with a 
concomitant reduction in phosphorylation $[60,62]$. In this respect, an intervention that increases $\mathrm{Rb}$ expression and/or increases $\mathrm{Rb}$ phosphorylation to normal physiological levels may provide some therapeutic benefit in HGPS.

\section{Apoptosis inhibitors}

Nuclear progerin accumulation leads to accelerated aging and increased apoptosis in individuals suffering from HGPS [59], and the same observation has been made in aging HGPS fibroblasts [63]. Theoretically, apoptosis inhibition (Table 3) may offer some means of extending the cellular lifespan of HGPS patients. However, the potential for increased risk of developing cancers and other related diseases would almost certainly limit such an approach.

\section{Inhibitors of translation and autophagy activators}

Rapamycin, an immunosuppressant drug, delays cellular senescence and organismal aging, abrogates nuclear blebbing, and stimulates degradation of progerin in HGPS cells $[64,65]$. This drug can selectively decrease progerin levels in progeria cells through a mechanism involving autophagic degradation [66]. Rapamycin treatment decreases the formation of nonsoluble aggregates of progerin and induces progerin elimination by autophagy in normal fibroblasts

Fig. 1 Key signaling pathways upstream of LMNA, which regulate its expression (listed mainly on the top of the figures), and the main molecules that interact with lamin $\mathrm{A} / \mathrm{C}$ protein (listed mainly in the nucleus). Labels denoting physiological processes in the cell are derived partially from the IPA listing under category "Top Functions and Diseases": Mechanical Stability of the Nucleus, Response to DNA Damage, Gene Transcription, Cell Cycle Progression. The meanings of the molecular symbols are described in the figure legend, which is part of the figure. Lines represent interactions between the molecules. They can have arrows, solid lines or nothing at the end, which represent directional action, inhibitory action, or just binding of two molecules, respectively. Red crossing lines in the nucleus represent a DNA double helix. Entrez Protein Names and their symbols used in figures, are in parenthesis: Catenin (cadherin-associated protein), beta $1,88 \mathrm{kDa}(\beta$-catenin), beta-transducin repeat containing $(\beta$ $\operatorname{Tr} C P$ ), eukaryotic translation initiation factor $4 \mathrm{E}$ binding protein 1 (eIF4EBP), AHR ligand, aromatic hydrocarbon (AHR Ligand), protein kinase B/Akt $(A K T)$, Adenomatous polyposis coli, APC ( $A P C)$, mitogenactivated protein kinase kinase kinase $5(A S K 1)$, adenosine 5 '-triphosphate $(A T P)$, axin 1 (Axin), barrier to autointegration factor 1 (BANF1), BCL2associated $\mathrm{X}$ protein $(B A X)$, B cell CLL/lymphoma 2 ( $B c l-2)$, beta-cateninLEF/TCF (Betacatenin/TCF), V-raf-1 murine leukemia viral oncogene homolog 1(c-Raf), cadherin $(E, N, P, V E)$, calcium-dependent protease, $\mathrm{M}$ calpain (Calpain), CASPASE-1 (Caspase), interleukin 1 converting enzyme (ICE), caspase 12 apoptosis-related cysteine peptidases (Caspases 2,3,6 and 12), casein kinase I (CKI), cyclic AMP (cAMP), eukaryotic translation initiation factor 2-alpha kinase $2(E I F 2 A K 2)$, eukaryotic translation initiation factors ( $e I F 4 A, 4 B, 4 G$, and $4 E$ ), emerin ( $E M D), \mathrm{p} 42 / \mathrm{p} 44$ MAP kinase $(E R K 1 / 2)$, filamentous actin ( $F$ Actin), PTK2 protein tyrosine kinase $2(F A K)$, FBJ murine osteosarcoma viral oncogene homolog (FOS), frizzled $(F Z, F Z D)$, MTOR-associated protein, LST8 homolog (S. cerevisiae) $(G B L)$, frequently rearranged in advanced T cell lymphomas $(G B P)$, glucagon receptor $(G C G R)$, growth hormone receptor $(G H R)$, growth
[64]. A safer alternative to rapamycin, rilmenidine, a centrally acting anti-hypertensive drug, was found to induce autophagy in cell culture via a pathway independent of the mammalian target of rapamycin [67]. As a natural alternative to the acid form of tretinoin (all-trans-retinoic acid), vitamin A has been found to induce autophagy. The essential oil produced from rose hip seeds is a natural source of tretinoin and promotes autophagosome maturation through a pathway independent from the classic nuclear hormone receptors $[68,69]$. Another natural autophagy activator is vitamin K2 [70]. A comprehensive list of known autophagy activators is listed in Table 4.

Several small molecules down-regulate lamin A/C protein via mechanisms of proteolysis. Doxorubicin (also known as adriamycin) is a topoisomerase II inhibitor used in anti-cancer therapy whereby it induces activation of caspases, leading to cleavage of lamin A/C [71]. Doxorubicin has also been identified in a high-content screen for inducers of autophagy [72]. Sangivamycin is a nucleoside analog that acts via activation of JNK and protein kinase $\mathrm{C}$ delta. In MCF-7/Adr cells, sangivamycin increases cleavage of human lamin A/C protein [73]. Tunicamycin and thapsigargin, endoplasmatic reticulum (ER) stress inducers, increase degradation of mouse LMNA protein via the activation of caspases [74]. Paclitaxel, a chemotherapeutic agent, induces cleavage of lamin $\mathrm{A} / \mathrm{C}$, enhanced by the synthetic peptides

factor receptor-bound protein 2 (GRB2), growth hormone ( $G H)$, glycogen synthase kinase $3(\mathrm{GSK} 3)$, hydrogen peroxide $\left(\mathrm{H}_{2} \mathrm{O}_{2}\right)$, HtrA serine pepti-

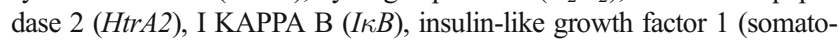
medin C) $(I G F-1)$, insulin-like growth factor 1 receptor (IGFIR), I kappa $\beta$-NF-kappa $\beta(I \kappa \beta-N F \kappa \beta)$, integrin-linked kinase $(I L K)$, mitogenactivated protein kinase 8 (JNK1), Jun proto-oncogene (JUN) lamin A/C ( $L M N A)$, LEM domain containing 3 (LEMD3), myelin-associated glycoprotein $(M A G)$, p53 binding protein homolog (mouse) (MDM2), methyl CpG-binding protein 2 (Rett syndrome) (MECP2), MAP kinases (MEK1/ 2, MKK4/7, MKK4/7), mechanistic target of rapamycin (serine/threonine kinase) mTORC1 (mTOR), V-myc myelocytomatosis viral oncogene homolog (avian) (MYC), NF-KAPPA B $(N F-k B)$, cyclin-dependent kinase inhibitor 2A (melanoma, p16, inhibits CDK4) (p14ARF), tumor protein p53 (p53, TP53), microtubule affinity-regulating kinase 2 (MAP), proliferating cell nuclear antigen $(P C N A)$, 1-phosphatidylinositol 3-kinase $(P I 3 K)$, peptidylprolyl cis/trans isomerase, NIMA-interacting 1 (PIN1), protein kinase $\mathrm{C}(P K C)$, phospholipase $\mathrm{C}$ gamma, $(P L C G)$, protein phosphatase type2a $(P P 2 A)$, peroxisome proliferator-activated receptor gamma $(P P A R G)$, protein kinase $\mathrm{C}$, alpha $(P R K C A)$, parathyroid hormone $(P T H)$, parathyroid hormone 1 receptor $(P T H 1 R)$, regulatory associated protein of MTOR, complex 1" (Raptor), p21 Ras (Ras), retinoblastoma 1 (RB1), Ras homolog enriched in brain (Rheb), (Src homology 2 domain containing)transforming protein 1 (SHC), SMAD family members (Smad3 and Smad4), V-src sarcoma (Schmidt-Ruppin A-2) viral oncogene homolog (avian) ( $S r c$ ), sterol regulatory element-binding transcription factor 1 (SREBF1), signal transducer and activator of transcription 4 (STAT4), Sad1 and UNC84 domain containing 1 (SUN1), Sad1 and UNC84 domain containing 2 (SUN2), spectrin repeat containing, nuclear envelope 1 (SYNE1), spectrin repeat containing, nuclear envelope 2 (SYNE2), TGF beta $(T g f \beta)$, thyroid hormone (T3), thyroid hormone receptor $(T R)$, polyubiquitin $(U b)$, von Hippel-Lindau tumor suppressor (VHL), WNT inhibitory factor $1($ WIFl) 


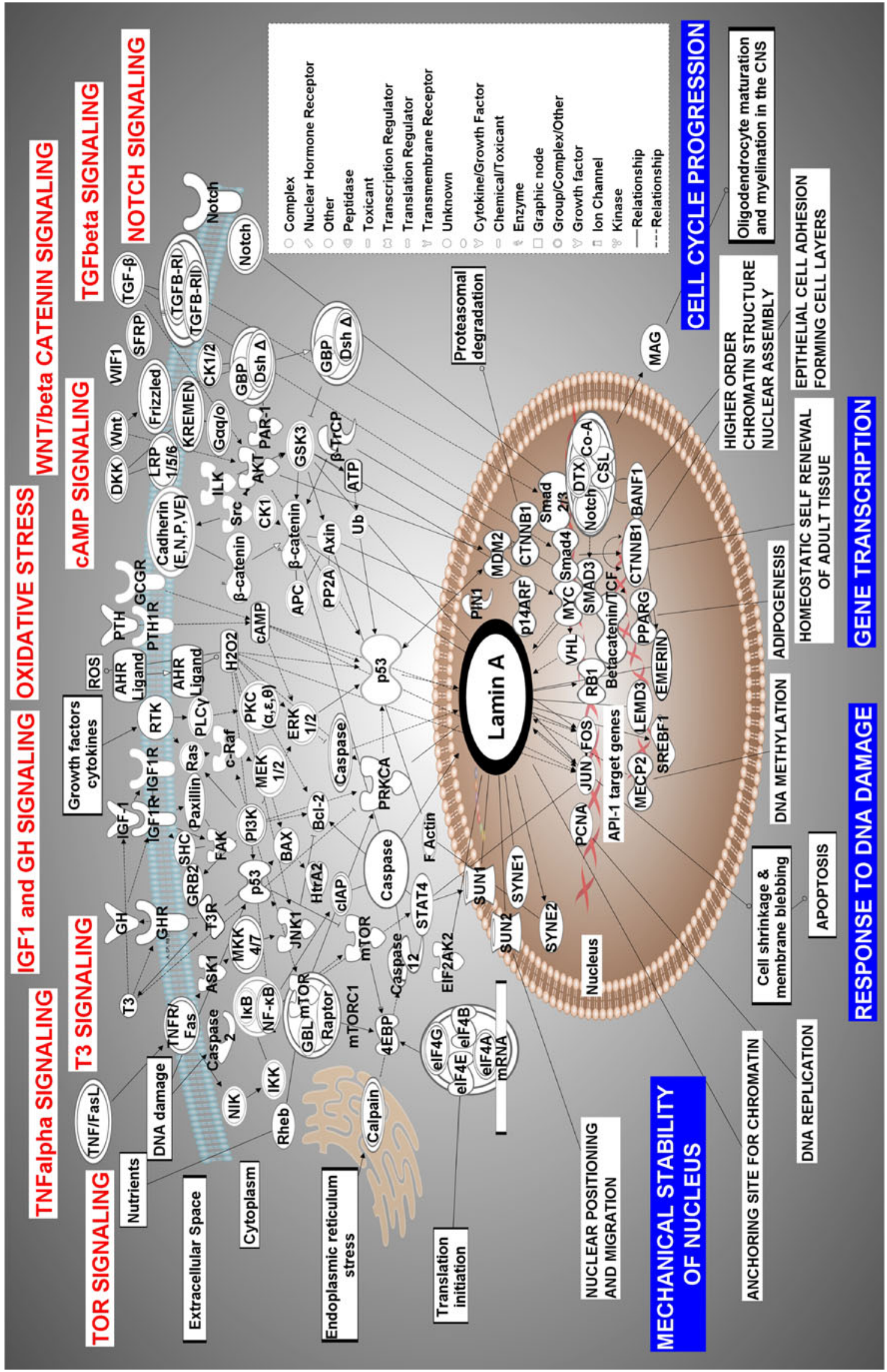


Table 1 Notch signaling inhibitors

\begin{tabular}{|c|c|c|c|c|}
\hline Compound & Mechanism of action & References & Side effects & SE references \\
\hline SEL-10 & $\begin{array}{l}\text { Ubiquitin-mediated } \\
\text { protein degradation }\end{array}$ & [119] & N/A & - \\
\hline L-658,458 (transition state mimic) & $\gamma$-Secretase-inhibitors & {$[120]$} & N/A & - \\
\hline $\begin{array}{c}N \text {-[N-(3,5-difluorophenacetyl)-L-alanyl }]- \\
S \text {-phenylglycine t-butyl ester (DAPT) }\end{array}$ & $\gamma$-Secretase-inhibitors & {$[120]$} & $\mathrm{N} / \mathrm{A}$, preclinical trials & {$[121]$} \\
\hline IL-X (cbz-IL-CHO) & $\gamma$-Secretase-inhibitors & {$[122]$} & Neutropenia, injection-site reactions & {$[123]$} \\
\hline WPE III-31-C & $\gamma$-Secretase-inhibitors & [124] & $\mathrm{N} / \mathrm{A}$ & - \\
\hline Compound E & $\gamma$-Secretase-inhibitors & [124] & N/A & - \\
\hline Sulfonamide & $\gamma$-Secretase-inhibitors & [124] & $\begin{array}{l}\text { Itching, burning, skin rash, redness, } \\
\text { swelling, urinary tract disorders, } \\
\text { haemopoietic disorders, porphyria, } \\
\text { and hypersensitivity reactions }\end{array}$ & {$[121,125]$} \\
\hline JLK6 & $\gamma$-Secretase-inhibitors & {$[124]$} & N/A & - \\
\hline Sulindac sulfide & $\gamma$-Secretase-inhibitors & [124] & $\begin{array}{l}\text { Stupor, diminished urine output and } \\
\text { hypotension }\end{array}$ & $\begin{array}{l}\text { Information from } \\
\text { the manufacturer }\end{array}$ \\
\hline Indomethacin & $\gamma$-Secretase-inhibitors & [124] & Nausea, dyspepsia, headache, dizziness & {$[121,126]$} \\
\hline Estrogen & $\begin{array}{l}\text { Inhibition of Notch } \\
\text { cleavage by } \gamma \text {-secretase }\end{array}$ & [127] & $\begin{array}{l}\text { Nausea, vomiting, withdrawal bleeding } \\
\text { (in females) }\end{array}$ & {$[121,128]$} \\
\hline RO4929097 & $\gamma$-Secretase-inhibitors & [129] & N/A, clinical trials & [121] \\
\hline
\end{tabular}

Smac/DIABLO [75]. It should be noted that HGPS is characterized by a large increase in the rate of apoptosis [63], and while application of apoptosis inducers in HGPS treatment is restricted, autophagy inducers can be considered safe, since they do not induce cell death [72].

\section{cAMP activators}

Several hormones have been identified as having effects on the expression, function, or phosphorylation of Lamin A. Hormones that increase cAMP levels (glucagon, calcitonin, vasopressin, and parathyroid hormone (PTH)) decrease lamin A protein phosphorylation of rat LMNA protein in renal medullary thick ascending limb cells [76]. cAMPdependent phosphorylation controls nuclear lamin associations, and aberrant phosphorylation could cause remodeling of the lamina [77]. Therefore, hormone modulation of lamin A phosphorylation with glucagon, calcitonin, vasopressin, or parathyroid hormone might be another way to alleviate laminopathy symptoms.

\section{Thyroid hormone supplementation}

Thyroid hormone (T3) decreases expression of mouse Lamin A mRNA in liver from mice exhibiting hypothyroidism [78]. An association has been reported between low levels of T3 and DeBarsi syndrome, an autosomal recessive syndrome characterized by a progeria-like appearance [79, 80]. The endocrine system is affected by aging, and while T3 has been associated with longevity, deficiencies in, or suboptimal levels of T3 are more common in older individuals, particularly women. Therefore, supplementation with

Table 2 Reactive oxygen species inhibitors

\begin{tabular}{|c|c|c|c|c|}
\hline Compound & Mechanism of action & References & Side effects & SE references \\
\hline $\begin{array}{l}\text { Tiron (4,5-dihydroxy-1, } \\
\text { 3-benzenedisulfonic acid) }\end{array}$ & ROS scavenger & {$[130,131]$} & N/A & - \\
\hline $\begin{array}{l}\text { Tempol (4-hydroxy-2,2,6, } \\
\text { 6-tetramethylpiperydine-1-oxyl) }\end{array}$ & $\begin{array}{l}\text { Superoxide dismutase } \\
\text { mimetic, antioxidant }\end{array}$ & {$[130,132]$} & N/A & - \\
\hline Glutathione (GSH) & $\begin{array}{l}\text { Antioxidant, reduces } \\
\text { disulfide bonds }\end{array}$ & {$[133]$} & N/A, clinical trials & {$[121]$} \\
\hline$N$-acetyl-cysteine (NAC) & $\begin{array}{l}\text { Antioxidant, breaks } \\
\text { disulfide bonds }\end{array}$ & {$[133]$} & $\begin{array}{l}\text { Reproductive toxicity, reduction } \\
\text { in fertility }\end{array}$ & {$[121,134]$} \\
\hline Vitamin C (ascorbic acid) & $\begin{array}{l}\text { Antioxidant, reducing } \\
\text { agent }\end{array}$ & {$[133]$} & $\begin{array}{l}\text { Nausea, vomiting, diarrhea, flushing } \\
\text { of the face, headache, fatigue, and } \\
\text { disturbed sleep }\end{array}$ & {$[135]$} \\
\hline Vitamin E (tocopherol) & Fat-soluble antioxidant & {$[133]$} & No significant side effects & {$[136,137]$} \\
\hline
\end{tabular}


Table 3 Apoptosis inhibitors

\begin{tabular}{|c|c|c|c|c|}
\hline Compound & Mechanism of action & References & Side effects & SE references \\
\hline $\begin{array}{l}\text { BBMP [5-(benzylsulfonyl)-4- } \\
\text { bromo-2-methyl-3(2H)- } \\
\text { yridazinone] }\end{array}$ & $\begin{array}{l}\text { Mitochondrial permeability transition pore } \\
\text { (PTP) inhibitor neuroprotective }\end{array}$ & [138] & N/A & - \\
\hline BI-6c9 & $\begin{array}{l}\text { Bid inhibitor, prevented loss of mitochondrial } \\
\text { outer membrane potential (MOMP) and } \\
\text { mitochondrial fission }\end{array}$ & {$[139-141]$} & N/A & - \\
\hline $\begin{array}{l}\text { BTZO-1 (1,3-benzothiazin-4-one } \\
\text { derivative) }\end{array}$ & $\begin{array}{l}\text { Activation of antioxidant response element } \\
\text { (ARE)-mediated gene expression }\end{array}$ & [142] & N/A & - \\
\hline Bongkrekic acid solution & $\begin{array}{l}\text { Inhibitors of mitochondrial permeability } \\
\text { transition (MPT) pore opening }\end{array}$ & [143-148] & $\mathrm{N} / \mathrm{A}$ & - \\
\hline Pifithrin- $\alpha$ hydrobromide & p53 Inhibitor & [149] & N/A & - \\
\hline NS3694 & Inhibitor of apoptosome formation & {$[150]$} & $\mathrm{N} / \mathrm{A}$ & - \\
\hline Z-ATAD-FMK & Inhibitor of apoptosome formation & {$[150]$} & N/A & - \\
\hline $\begin{array}{l}N \text {-benzylisatin sulfonamide } \\
\text { analogues }\end{array}$ & Caspase-3 inhibitor & {$[151]$} & N/A & - \\
\hline 5-Dialkylaminosulfonylisatins & Caspase- 3 and 7 inhibitor & [152] & N/A & - \\
\hline $16 \mathrm{~F} 16$ & Protein disulfide isomerase (PDI) inhibitor & [153] & $\mathrm{N} / \mathrm{A}$ & - \\
\hline $16 \mathrm{~F} 16 \mathrm{~A}$ & Protein disulfide isomerase (PDI) inhibitor & [153] & N/A & - \\
\hline 16F16A-DC & Protein disulfide isomerase (PDI) inhibitor & [153] & N/A & - \\
\hline Thiomuscimol & Protein disulfide isomerase (PDI) inhibitor & [153] & N/A & - \\
\hline Cystamine & Protein disulfide isomerase (PDI) inhibitor & [153] & $\begin{array}{l}\text { Loss of appetite, diarrhea, } \\
\text { drowsiness, lack of energy, } \\
\text { nausea, stomach pain, } \\
\text { unpleasant breath odor, } \\
\text { vomiting }\end{array}$ & {$[154,155]$} \\
\hline Pifithrin- $\alpha$ & p53 Inhibitor & {$[156,157]$} & N/A & - \\
\hline Pifithrin- $\mu$ (PFTmu, 1) & Inhibits p53 binding to mitochondria & {$[158]$} & $\mathrm{N} / \mathrm{A}$ & - \\
\hline S-15176 difumarate salt & Inhibits mitochondrial permeability transition & [159] & N/A & - \\
\hline IDN-6556 & Inhibitor of caspase 3 & {$[160]$} & $\mathrm{N} / \mathrm{A}$ & - \\
\hline
\end{tabular}

Table 4 Autophagy activators

\begin{tabular}{|c|c|c|c|c|}
\hline Compound & Mechanism of action & References & Side effects & SE references \\
\hline $\begin{array}{l}\text { 10-(6'-Ubiquinonyl)decyltriphenyl } \\
\text { phosphonium (MitoQ) }\end{array}$ & $\begin{array}{l}\text { Redox-active ubiquinone } \\
\text { that accumulates in } \\
\text { mitochondria }\end{array}$ & {$[161]$} & N/A, clinical trial & {$[121]$} \\
\hline $\begin{array}{l}\text { 1-Alpha, } 25 \text {-dihydroxy } \\
\text { vitamin D3 }\end{array}$ & $\begin{array}{l}\mathrm{Ca}++ \text { regulator } \\
\text { VDR modulator }\end{array}$ & {$[162]$} & Hypercalcemia & {$[121,163]$} \\
\hline 2-Deoxyglucose (2-DG) & $\begin{array}{l}\text { Binds to hexokinase, } \\
\text { marker for tissue } \\
\text { glucose use }\end{array}$ & {$[164,165]$} & $\mathrm{N} / \mathrm{A}$, clinical trials & {$[121]$} \\
\hline 5-Fluorouracil & $\begin{array}{l}\text { Inhibits thymidylate } \\
\text { synthase }\end{array}$ & {$[166]$} & $\begin{array}{l}\text { Nausea, vomiting, diarrhea, } \\
\text { gastrointestinal ulceration } \\
\text { and bleeding, and bone marrow } \\
\text { depression }\end{array}$ & {$[121,167]$} \\
\hline 6-Hydroxydopamine & Neurotransmitter & {$[168]$} & N/A & {$[121]$} \\
\hline A23187 & ER stress & [169] & N/A & [121] \\
\hline Amiodarone & Anti-arrhythmic drug & {$[170]$} & $\begin{array}{l}\text { Abnormal taste or smell, constipation, } \\
\text { decreased sexual interest, dizziness, } \\
\text { dry eyes, flushing of the face, } \\
\text { general body discomfort, headache, } \\
\text { loss of appetite, nausea, tiredness, } \\
\text { trouble sleeping, vomiting }\end{array}$ & {$[121,171]$} \\
\hline
\end{tabular}


Table 4 (continued)

\begin{tabular}{|c|c|c|c|c|}
\hline Compound & Mechanism of action & References & Side effects & SE references \\
\hline Ammonia & $\begin{array}{l}\text { By-product of amino acid } \\
\text { metabolism }\end{array}$ & {$[172]$} & N/A & {$[121]$} \\
\hline Arsenic trioxide & $\begin{array}{l}\text { MEK/ERK pathway } \\
\text { Beclin } 1 \text { or Atg } 7 \text { targets } \\
\text { Induces } \mathrm{XCP} \text {, not XCA }\end{array}$ & {$[173,174]$} & $\begin{array}{l}\text { QT interval prolongation, arrhythmia, } \\
\text { tachycardia, fatigue, fever, edema, } \\
\text { convulsions, muscle weakness, } \\
\text { confusion }\end{array}$ & {$[121,175]$} \\
\hline Aspirin & $\begin{array}{l}\text { Inhibitor of mTOR and } \\
\text { activator of AMP-activated } \\
\text { protein kinase }\end{array}$ & {$[176]$} & $\begin{array}{l}\text { Black, bloody, or tarry stools, } \\
\text { coughing up blood or vomit, nausea, } \\
\text { stomach pain, fever, upset stomach, } \\
\text { heartburn, headache, tinnitus }\end{array}$ & {$[121,177]$} \\
\hline Atorvastatin & AMP-activated protein kinase & [178] & $\begin{array}{l}\text { Muscle pain, memory problems, fever, } \\
\text { unusual tiredness, dark-colored } \\
\text { urine, swelling, thirst, dry mouth, } \\
\text { nausea }\end{array}$ & {$[121,179]$} \\
\hline $\begin{array}{l}\text { Aurintricarboxylic } \\
\text { acid (ATA) }\end{array}$ & ERK1/2 activation & {$[180]$} & $\begin{array}{l}\text { Adverse effect for focal nodular } \\
\text { hyperplasia }\end{array}$ & $\begin{array}{l}\text { Information from } \\
\text { the manufacturer }\end{array}$ \\
\hline AZD-8055 & $\begin{array}{l}\text { mTOR ATP-competitive } \\
\text { inhibitor }\end{array}$ & [181] & N/A, clinical trials & {$[121]$} \\
\hline Benzaldehyde & Formation of autophagosome & [182] & N/A, clinical trials & [121] \\
\hline Bortezomib & $\begin{array}{l}\text { PSMB5 inhibitor, } 26 \mathrm{~S} \\
\text { proteasome inhibitor }\end{array}$ & {$[183,184]$} & $\begin{array}{l}\text { Nerve problems, dry cough, trouble } \\
\text { breathing, headache, vision } \\
\text { problems, bleeding, fever, fast or } \\
\text { slow heart rate, nausea, diarrhea }\end{array}$ & {$[121,185]$} \\
\hline Brefeldin A & $\begin{array}{l}\text { Endoplasmic reticulum } \\
\text { (ER)-to-golgi traffic } \\
\text { inhibitor }\end{array}$ & [186] & N/A & - \\
\hline $\mathrm{Ca} 2+$ & Via $\mathrm{Ca}++$ channels & $\begin{array}{l}\text { ref in }[187, \\
188]\end{array}$ & N/A & - \\
\hline Capsaicin & $\begin{array}{l}\text { Through the AMPK } \alpha \text {-mTOR } \\
\text { signaling pathway and the } \\
\text { accumulation of p53 in the } \\
\text { nucleus }\end{array}$ & [189] & $\begin{array}{l}\text { Nausea, vomiting, abdominal pain } \\
\text { burning diarrhea, eye exposure }\end{array}$ & {$[190]$} \\
\hline $\begin{array}{l}\text { Carbamazepine } \\
\text { (CBZ) }\end{array}$ & $\begin{array}{l}\text { Radiation protector and } \\
\text { mitigator } \\
\text { Anti-epilepsy and mood- } \\
\text { stabilizing drug }\end{array}$ & {$[191,192]$} & $\begin{array}{l}\text { Dizziness, drowsiness, dry mouth, } \\
\text { nausea, unsteadiness, vomiting }\end{array}$ & {$[121,193]$} \\
\hline Ceramide & Protein kinase B inhibition & {$[194,195]$} & N/A & - \\
\hline Cetuximab & EGFR inhibitor & [196] & $\begin{array}{l}\text { Changes in fingernails or toenails, } \\
\text { constipation, cough, diarrhea, } \\
\text { dry mouth, headache, indigestion, } \\
\text { nausea, pain, swelling, stomach } \\
\text { pain or upset, vomiting, weakness, } \\
\text { weight loss }\end{array}$ & {$[121,197]$} \\
\hline Cisplatin & DNA-damaging agent & [198] & $\begin{array}{l}\text { Diarrhea, loss of appetite, nausea, } \\
\text { vomiting, weakness }\end{array}$ & {$[121,199]$} \\
\hline Chlorpromazine & $\begin{array}{l}\text { Used to treat the symptoms } \\
\text { of schizophrenia }\end{array}$ & [200] & $\begin{array}{l}\text { Agitation, constipation, dizziness, } \\
\text { drowsiness, dry mouth, enlarged } \\
\text { pupils, jitteriness, nausea, stuffy nose }\end{array}$ & {$[121,201]$} \\
\hline Cholecalciferol & Vitamin D & [202] & Constipation & {$[121,203]$} \\
\hline Clonidine & G(i) signaling activator & [204] & $\begin{array}{l}\text { Anxiety, confusion, constipation, } \\
\text { dizziness, drowsiness, dry mouth, } \\
\text { general weakness, nausea, ringing in } \\
\text { the ears, sweating, tiredness, vomiting }\end{array}$ & {$[121,205]$} \\
\hline Cucurbitacin & STAT3 inhibitor & [206] & $\mathrm{N} / \mathrm{A}$ & - \\
\hline $\begin{array}{l}\text { Deforolimus, } \\
\text { Ridaforolimus }\end{array}$ & MTOR inhibitor & [207] & $\begin{array}{l}\text { Tiredness and feeling weak, loss of } \\
\text { appetite, sore mouth and throat, rash, } \\
\text { a drop in blood cells causing an } \\
\text { increased risk of infection, tiredness } \\
\text { and breathlessness, diarrhea, fatigue, } \\
\text { anorexia }\end{array}$ & {$[205,208]$} \\
\hline
\end{tabular}


Table 4 (continued)

\begin{tabular}{|c|c|c|c|c|}
\hline Compound & Mechanism of action & References & Side effects & SE references \\
\hline $\begin{array}{l}\text { Delta-9- } \\
\text { tetrahydrocannabinol } \\
\text { (THC) }\end{array}$ & $\begin{array}{l}\text { Psychoactive ingredient in } \\
\text { marijuana }\end{array}$ & [209] & CNS adverse & $\begin{array}{l}\text { Information from } \\
\text { the manufacturer }\end{array}$ \\
\hline Dexamethasone & Glucocorticoid hormones & [210] & $\begin{array}{l}\text { Problems with vision, swelling, } \\
\text { pancreatitis, depression, bloody } \\
\text { stools, low potassium, high blood } \\
\text { pressure }\end{array}$ & {$[121,211]$} \\
\hline $\begin{array}{l}\text { Digeranyl } \\
\text { bisphosphonate }\end{array}$ & $\begin{array}{l}\text { Inhibitor of geranylgeranyl } \\
\text { diphosphate synthase } \\
\text { (GGDPS) }\end{array}$ & [212] & $\begin{array}{l}\text { Renal toxicity, acute-phase reactions, } \\
\text { gastrointestinal toxicity, and } \\
\text { osteonecrosis of the jaw }\end{array}$ & [213] \\
\hline D-Glucose & ER stress & {$[165]$} & N/A, clinical trials & [121] \\
\hline $\begin{array}{l}\text { Docosahexaenoic } \\
\text { acid (DHA) }\end{array}$ & $\begin{array}{l}\text { Through p53/AMPK/mTOR } \\
\text { signaling }\end{array}$ & [214] & $\begin{array}{l}\text { Back pain, flu, infection, pain, angina } \\
\text { pectoris, dyspepsia, eructation, rash, } \\
\text { taste perversion }\end{array}$ & {$[121,215]$} \\
\hline Doxorubicin & $\begin{array}{l}\text { Antitumor antibiotic, } \\
\text { chemotherapy drug }\end{array}$ & {$[216]$} & $\begin{array}{l}\text { Cardiac failure, arrhythmias, } \\
\text { thrombocytopenia, leukopenia, } \\
\text { nausea, vomiting, ulceration in the } \\
\text { gastrointestinal tract, hyperuricemia, } \\
\text { nephropathy }\end{array}$ & [217] \\
\hline Epothilone B & Microtubule-stabilizing agent & [218] & N/A, clinical trials & {$[121]$} \\
\hline $\begin{array}{l}\text { Esomeprazole } \\
\text { magnesium }\end{array}$ & Proton-pump inhibitor & [219] & Headache, diarrhea, abdominal pain & $\begin{array}{l}\text { Information from } \\
\text { the manufacturer }\end{array}$ \\
\hline Etoposide & Topoisomerase inhibitor & {$[220]$} & $\begin{array}{l}\text { Loss of appetite, back pain, blue or } \\
\text { purple discoloration of the skin, } \\
\text { diarrhea, hair loss, increased } \\
\text { sweating, nausea, tightness in } \\
\text { throat, voice changes, vomiting }\end{array}$ & {$[121,221]$} \\
\hline Everolimus & $\begin{array}{l}\text { MTOR inhibitor; FKBP1A } \\
\text { binder }\end{array}$ & [222] & $\begin{array}{l}\text { Stomatitis, rash, diarrhea, fatigue, } \\
\text { edema, abdominal pain, nausea, } \\
\text { fever, andheadache }\end{array}$ & [223] \\
\hline Fluspirilene & $\begin{array}{l}\text { Diphenylbutylpiperidine } \\
\text { antipsychotic drug }\end{array}$ & {$[200,224]$} & $\begin{array}{l}\text { Sleepiness, movement disorders, pain } \\
\text { where the injection is given }\end{array}$ & {$[225,226]$} \\
\hline GNE477 & Dual PI3K/mTOR inhibitors & [181] & $\mathrm{N} / \mathrm{A}$ & - \\
\hline Glucose-6-phosphate & Glucose homeostasis & [227] & N/A & - \\
\hline Gossypol & Natural phenol BCL2 inhibitor & [228] & N/A, clinical trials & [121] \\
\hline GSK2126458 & Dual PI3K/mTOR inhibitors & [181] & N/A, clinical trials & [121] \\
\hline Hydrogen peroxide & $\begin{array}{l}\text { Reactive oxygen species, } \\
\text { signaling molecule }\end{array}$ & [229] & $\begin{array}{l}\text { Nausea and vomiting, burns in the } \\
\text { mouth, throat, esophagus, and } \\
\text { stomach, bleeding in the stomach, } \\
\text { inflammation of the intestines }\end{array}$ & $\begin{array}{l}\text { Information from } \\
\text { the manufacturer }\end{array}$ \\
\hline INK 128 & mTOR ATP-competitive inhibitor & {$[181]$} & $\mathrm{N} / \mathrm{A}$, clinical trials & [121] \\
\hline Imatinib & $\begin{array}{l}\text { PDGFRA, PDGFRB inhibitor } \\
\text { BCR/ABL tyrosine kinase } \\
\text { inhibitors }\end{array}$ & {$[230]$} & $\begin{array}{l}\text { Nausea, vomiting, diarrhea, loss } \\
\text { of appetite, dry skin, hair loss, } \\
\text { swelling, and muscle cramps }\end{array}$ & {$[121,231]$} \\
\hline Ionomycin & Potent a $\mathrm{Ca}++$ ionophore & [232] & $\begin{array}{l}\text { Changes in lung function, } \\
\text { i.e., pneumoconiosis }\end{array}$ & $\begin{array}{l}\text { Information from } \\
\text { the manufacturer }\end{array}$ \\
\hline Lactacystin & $\begin{array}{l}\text { Enhanced degradation of } \\
\text { aggregated proteins }\end{array}$ & {$[187]$} & N/A & - \\
\hline Lanosterol & $\begin{array}{l}\text { Regulates mitochondrial } \\
\text { function }\end{array}$ & {$[233]$} & $\begin{array}{l}\text { Changes in lung function, } \\
\text { i.e., pneumoconiosis }\end{array}$ & $\begin{array}{l}\text { Information from } \\
\text { the manufacturer }\end{array}$ \\
\hline L-Arginine & Amino acid & [234] & N/A, clinical trials & {$[121]$} \\
\hline Leupeptin & Protease inhibitor & [235] & $\mathrm{N} / \mathrm{A}$ & - \\
\hline $\begin{array}{l}\text { Lipopolysaccharide } \\
\text { (LPS) }\end{array}$ & Bacterial endotoxin & [236] & $\begin{array}{l}\text { LPS is an adjuvant for both humoral } \\
\text { and cell-mediated immunity. It } \\
\text { augments the immune response to } \\
\text { both protein and polysaccharide } \\
\text { antigens. It is too toxic and }\end{array}$ & $\begin{array}{l}\text { Information from } \\
\text { the manufacturer }\end{array}$ \\
\hline
\end{tabular}


Table 4 (continued)

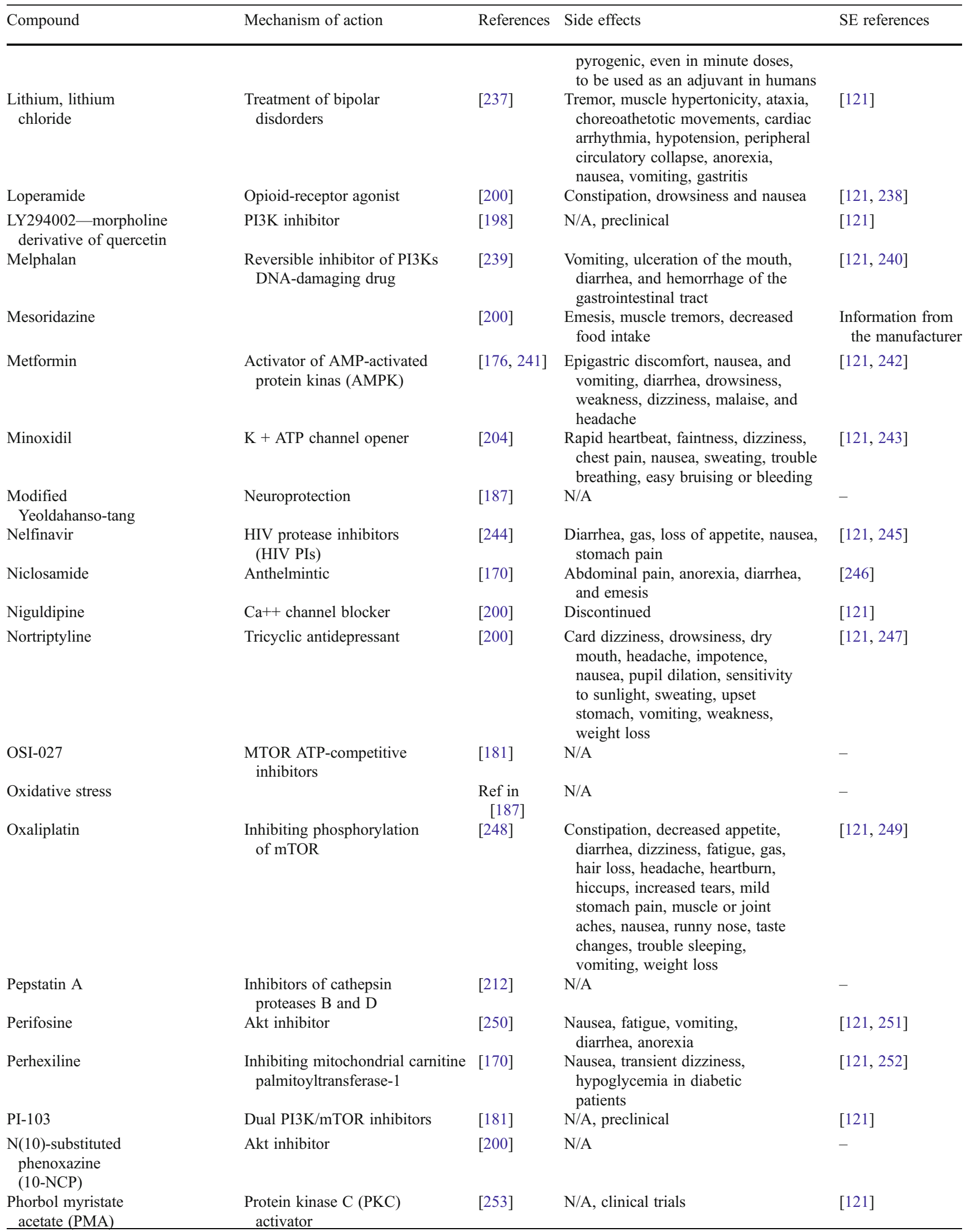


Table 4 (continued)

\begin{tabular}{|c|c|c|c|c|}
\hline Compound & Mechanism of action & References & Side effects & SE references \\
\hline $\begin{array}{l}\text { Phosphatidylinositol- } \\
\text { 3-phosphate }\end{array}$ & Membrane phospholipid & {$[254]$} & N/A & - \\
\hline Pimozide & $\begin{array}{l}\text { Antipsychotic drug of the } \\
\text { diphenylbutylpiperidine class }\end{array}$ & {$[200]$} & $\begin{array}{l}\text { Constipation, drowsiness, dry } \\
\text { mouth, restlessness }\end{array}$ & [255] \\
\hline Promazine & $\begin{array}{l}\text { Phenothiazine class of } \\
\text { antipsychotics }\end{array}$ & {$[200]$} & $\begin{array}{l}\text { Extrapyramidal symptoms, } \\
\text { drowsiness, weight gain, dry } \\
\text { mouth, constipation, endocrine } \\
\text { effects, hemolytic anemia }\end{array}$ & {$[121,256]$} \\
\hline Promethazine & $\begin{array}{l}\text { Antihistamine of the } \\
\text { phenothiazine family }\end{array}$ & {$[200]$} & $\begin{array}{l}\text { Mild depression of CNS and } \\
\text { cardiovascular system, profound } \\
\text { hypotension, respiratory depression, } \\
\text { unconsciousness }\end{array}$ & {$[121,257]$} \\
\hline Propranolol & Cardiodepressant & {$[258]$} & $\begin{array}{l}\text { Bradycardia, cardiac failure, } \\
\text { hypotension, and bronchospasm }\end{array}$ & {$[121,259]$} \\
\hline Rapamycin (Sirolimus) & Antibiotic & {$[181,260]$} & $\mathrm{N} / \mathrm{A}$, clinical trials & {$[121]$} \\
\hline Reactive oxygen species & DNA damage & [206] & N/A & -_ \\
\hline Resveratrol & Nature phytoalexin & [261] & N/A, clinical trials & [121] \\
\hline Rilmenidine & Anti-hypertensive drug & {$[67]$} & $\begin{array}{l}\text { Asthenia, palpitations, insomnia, } \\
\text { drowsiness, fatigue on exercise, } \\
\text { epigastric pain, dryness of the } \\
\text { mouth, diarrhea, skin rash }\end{array}$ & [262] \\
\hline Rottlerin & Kinase inhibitor & {$[170]$} & N/A & - \\
\hline Saquinavir & $\begin{array}{l}\text { Antiretroviral protease } \\
\text { inhibitors }\end{array}$ & [263] & $\begin{array}{l}\text { Anxiety, blurred vision, body fat } \\
\text { changes, changes in sexual desire, } \\
\text { constipation, diarrhea, dizziness, dry } \\
\text { lips or skin, gas, headache, } \\
\text { heartburn, mouth sores, nausea, } \\
\text { night sweats, sleeplessness, stomach } \\
\text { discomfort, taste changes, tenderness } \\
\text { or bleeding of the gums, tiredness, } \\
\text { vomiting, warts, weight gain }\end{array}$ & {$[121,264]$} \\
\hline Sodium selenite & $\begin{array}{l}\text { Activation of DAPK via PP2A- } \\
\text { mediated dephosphorylation } \\
\text { at } \operatorname{Ser}(308)\end{array}$ & {$[265]$} & $\mathrm{N} / \mathrm{A}$, clinical trials & [121] \\
\hline Sorafenib & Multikinase inhibitor & {$[266]$} & Diarrhea and dermatologic events & {$[121,267]$} \\
\hline Spermidine & $\begin{array}{l}\text { Inhibition of mTOR or } \\
\text { activation of AMPK }\end{array}$ & {$[261,268]$} & N/A & - \\
\hline Staurosporine & Kinase inhibitor & [269] & N/A & - \\
\hline Superoxide & Oxidative stress & [270] & N/A & - \\
\hline Tamoxifen & Anti-estrogen & [271] & $\begin{array}{l}\text { Hot flashes, hypercalcemia, peripheral } \\
\text { edema, distaste for food, pruritus } \\
\text { vulvae, depression, dizziness, } \\
\text { lightheadedness, headache }\end{array}$ & {$[121,272]$} \\
\hline Temsirolimus & MTOR binder & {$[271]$} & $\begin{array}{l}\text { Hypersensitivity, hyperglycemia, } \\
\text { interstitial lung disease, } \\
\text { hyperlipidemia, bowel perforation, } \\
\text { renal failure }\end{array}$ & {$[121,273]$} \\
\hline Thapsigargin & $\begin{array}{l}\text { Non-competitive inhibitor of } \\
\text { sarco/endoplasmic reticulum } \\
\mathrm{Ca}++ \text { ATPase }\end{array}$ & {$[232]$} & N/A, preclinical & [121] \\
\hline Thioguanine & $\begin{array}{l}\text { Nucleoside analog induces DNA } \\
\text { mismatch repair }\end{array}$ & {$[274]$} & $\begin{array}{l}\text { Nausea, vomiting, malaise, } \\
\text { hypotension, and diaphoresis }\end{array}$ & {$[2,275]$} \\
\hline Thioridazine & $\begin{array}{l}\text { Antipsychotic drug belonging to } \\
\text { the phenothiazine drug }\end{array}$ & {$[200]$} & $\begin{array}{l}\text { Agitation, blurred vision, confusion, } \\
\text { constipation, difficulty breathing, } \\
\text { dilated or constricted pupils, } \\
\text { diminished flow of urine, dry mouth, } \\
\text { dry skin, excessively high or low } \\
\text { body temperature, extremely low } \\
\text { blood pressure, fluid in the lungs, } \\
\text { heart abnormalities, inability to }\end{array}$ & {$[121,276]$} \\
\hline
\end{tabular}


Table 4 (continued)

\begin{tabular}{|c|c|c|c|c|}
\hline Compound & Mechanism of action & References & Side effects & SE references \\
\hline & & & $\begin{array}{l}\text { urinate, intestinal blockage, nasal } \\
\text { congestion, restlessness, sedation, } \\
\text { seizures, shock }\end{array}$ & \\
\hline Trehalose & $\begin{array}{l}\text { Natural disaccharide implicated } \\
\text { in anhydrobiosis }\end{array}$ & [277] & N/A, clinical trials & [121] \\
\hline Tretinoin & Retinoic acid & {$[68,69]$} & Headache, fever, weakness, and fatigue & {$[121,278]$} \\
\hline Triflupromazine & $\begin{array}{l}\text { Antipsychotic medication of } \\
\text { the phenothiazine class }\end{array}$ & [200] & $\begin{array}{l}\text { Agitation, convulsions, difficulty } \\
\text { breathing, difficulty swallowing, dry } \\
\text { mouth, extreme sleepiness, fever, } \\
\text { intestinal blockage, irregular heart } \\
\text { rate, low blood pressure, and } \\
\text { restlessness }\end{array}$ & $\begin{array}{l}\text { Information from } \\
\text { the manufacturer }\end{array}$ \\
\hline $\begin{array}{l}\text { UCN-01 } \\
\text { (7-hydrostaurosporine) }\end{array}$ & Akt inhibitor & [279] & N/A & - \\
\hline Valinomycin & $\mathrm{K}(+)$-selective ionophore & {$[280]$} & N/A & - \\
\hline Valproic acid & $\begin{array}{l}\text { Anti-epilepsy and mood- } \\
\text { stabilizing drug }\end{array}$ & [192] & $\begin{array}{l}\text { Constipation, diarrhea, dizziness, } \\
\text { drowsiness, headache, increased or } \\
\text { decreased appetite, mild hair loss, } \\
\text { nausea, sore throat, stomach pain or } \\
\text { upset, trouble sleeping, vomiting, } \\
\text { weakness, weight gain }\end{array}$ & {$[121,281]$} \\
\hline Verapamil & Cardiodepressant & {$[258]$} & $\begin{array}{l}\text { Chest pain, arrhythmia, heart attacks, } \\
\text { significant water retention, dizziness }\end{array}$ & [282] \\
\hline $\begin{array}{l}\text { Vitamin K2 } \\
\text { (menaquinone-4) }\end{array}$ & Vitamin & {$[70]$} & N/A, clinical trials & {$[121]$} \\
\hline Vorinostat & HDAC6 inhibitor & [283] & $\begin{array}{l}\text { Diarrhea, nausea, anorexia, weight } \\
\text { decrease, vomiting, constipation, } \\
\text { thrombocytopenia, anemia }\end{array}$ & {$[121,284]$} \\
\hline VX-680 & Aurora-B kinase inhibitor & {$[285]$} & N/A, clinical trials & {$[121]$} \\
\hline WJD008 & Dual PI3K/mTOR inhibitors & {$[181]$} & N/A & - \\
\hline Y 27632 & ROCK inhibitors & [286] & Discontinued for hypertension & {$[121]$} \\
\hline zVAD & Pan-caspase inhibitor & {$[287,288]$} & N/A & - \\
\hline Zoledronate & $\begin{array}{l}\text { Inhibitor of farnesyl diphosphate } \\
\text { synthase (FDPS) }\end{array}$ & [212] & $\begin{array}{l}\text { Hypocalcemia, hypophosphatemia, } \\
\text { hypomagnesemia }\end{array}$ & {$[121,289]$} \\
\hline 68093 & ND & [290] & N/A & - \\
\hline 169676 & Eg5 inhibitor possibly & [290] & N/A & - \\
\hline 175493 & ND & [290] & N/A & - \\
\hline 363998 & ND & [290] & N/A & - \\
\hline 4-Piperidinone & Mitotic inhibition & [290] & N/A & - \\
\hline Aatiram & ND & [290] & $\begin{array}{l}\text { Adverse effect for allergic contact } \\
\text { dermatitis }\end{array}$ & $\begin{array}{l}\text { [121] Information } \\
\text { from the } \\
\text { manufacturer }\end{array}$ \\
\hline Acridine Yellow & DNA damage & [290] & N/A & - \\
\hline Bafilomycin A1 & Vacuolar ATPase inhibitor & [290] & N/A & - \\
\hline Bepridil & $\mathrm{Ca}++$ channels & [290] & $\begin{array}{l}\text { Dizziness, lightheadedness, diarrhea, } \\
\text { heartburn, nausea, blurred vision, } \\
\text { muscle cramps, headache, fatigue, } \\
\text { drowsiness, ringing in the ears, } \\
\text { flushing, trembling, or shaking hands }\end{array}$ & [291] \\
\hline Diosgenin & $\mathrm{BK} \mathrm{Ca++} \mathrm{channel}$ & {$[290]$} & N/A & - \\
\hline E6 Berbamine & Calmodulin inhibitor & [290] & N/A & - \\
\hline Fluspiriline & Potassium channels & [290] & $\begin{array}{l}\text { Gynecomastia, impotence, } \\
\text { agranulocytosis, galactorrhea, } \\
\text { tachycardia, blurred vision, } \\
\text { pyrexia, cataracts, dyskinesia }\end{array}$ & {$[225]$} \\
\hline Loperamide & $\mathrm{Ca}++$ channels & [290] & $\begin{array}{l}\text { Constipation, decreased urination, red, } \\
\text { swollen, blistered, or peeling skin, } \\
\text { stomach bloating, swelling, or pain }\end{array}$ & {$[121,238]$} \\
\hline
\end{tabular}


Table 4 (continued)

\begin{tabular}{|c|c|c|c|c|}
\hline Compound & Mechanism of action & References & Side effects & SE references \\
\hline Monensin & $\mathrm{Na}+$ ionophore & [290] & Adverse effect for myoglobinuria & $\begin{array}{l}\text { [121] Information } \\
\text { from the } \\
\text { manufacturer }\end{array}$ \\
\hline Nigericin & Induces intracellular acidification & {$[290]$} & N/A & - \\
\hline Purpurine & ND & [290] & N/A & - \\
\hline Pyridine derivative & ND & [290] & N/A & - \\
\hline Rottlerin & PKC delta inhibitor & [290] & $\begin{array}{l}\text { Suppressed cell/tissue growth or } \\
\text { development }\end{array}$ & \\
\hline Stannane & Aquaporin inhibitor & {$[290]$} & $\mathrm{N} / \mathrm{A}$ & - \\
\hline Tetrandrine & $\mathrm{Ca}++$ channels & [290] & Immunosuppressant activity & [292] \\
\hline Tetrocarcin A & BCL-2 inhibitor/ER stress & [290] & N/A & - \\
\hline Thalicarpine & $\begin{array}{l}\text { p-Glycoprotein inhibitor/DNA } \\
\text { damage }\end{array}$ & [290] & N/A & - \\
\hline Trichostatin-A & Histone deacetylase inhibitor & [290] & N/A, clinical trials & [121] \\
\hline Trifluoperazine & Calmodulin inhibitor possibly & [290] & $\begin{array}{l}\text { Agitation, constipation, dizziness, } \\
\text { drowsiness, dry mouth, enlarged } \\
\text { pupils, headache, jitteriness, loss } \\
\text { of appetite, nausea, stuffy nose, } \\
\text { tiredness }\end{array}$ & [293] \\
\hline Tyrphostine 9 & PDGF-R tyrosine kinase inhibitor & {$[290]$} & $\mathrm{N} / \mathrm{A}$ & - \\
\hline
\end{tabular}

Table 5 Methyl transferase inhibitors

\begin{tabular}{|c|c|c|c|c|}
\hline Compound & Mechanism of action & References & Side effects & SE references \\
\hline 5-Azacytidine (Vidaza) & $\begin{array}{l}\text { Nucleoside inhibitors-[DNMT] } \\
\text { enzyme trapping and degradation }\end{array}$ & [294] & $\begin{array}{l}\text { Nausea, anemia, thrombocytopenia, } \\
\text { vomiting, fever, diarrhea, neutropenia }\end{array}$ & {$[295]$} \\
\hline $\begin{array}{l}\text { 5-Azadeoxycytidine } \\
\text { (decitabine) }\end{array}$ & $\begin{array}{l}\text { Nucleoside inhibitors-[DNMT] } \\
\text { enzyme trapping and degradation }\end{array}$ & [294] & $\begin{array}{l}\text { Constipation, cough, diarrhea, dizziness, } \\
\text { hair loss, headache, joint or muscle pain, } \\
\text { loss of appetite, nausea, stomach pain or } \\
\text { upset, trouble sleeping, vomiting }\end{array}$ & [296] \\
\hline Zebularine & Nucleoside inhibitors & [294] & N/A & - \\
\hline Procaine & Mask DNMT target sequences & [294] & $\begin{array}{l}\text { Chest pain or slow, irregular heartbeats, } \\
\text { dizziness, anxiety, nausea, convulsions }\end{array}$ & [297] \\
\hline $\begin{array}{l}\text { Epigallocatechin- } \\
\text { 3-gallate (EGCG) }\end{array}$ & Green tea's active ingredient & [294] & Nausea and indigestion, neural tube defect & [298] \\
\hline RG108 & DNA methyltransferase inhibitor & [294] & N/A & - \\
\hline Procainamide & $\begin{array}{l}\text { Drug for treatment of cardiac } \\
\text { arrhytmia, non-covalent inhibitor } \\
\text { of } \mathrm{Na}+\text { channel }\end{array}$ & [299] & $\begin{array}{l}\text { Rash, myalgia, fever. Treatment with } \\
\text { procainamide can cause antibody } \\
\text { production against cellular components, } \\
\text { accounting for the systemic lupus } \\
\text { erythematosus-like adverse reactions }\end{array}$ & {$[300]$} \\
\hline Parthenolide & $\begin{array}{l}\text { Modulation of NF-kappa } \beta \text { activity, } \\
\text { microtubule interfering }\end{array}$ & {$[301]$} & Vomiting, abdominal pain, and indigestion & {$[302]$} \\
\hline Curcumin & $\begin{array}{l}\text { Natural phenol, gives yellow color to } \\
\text { turmeric, interferes with NF-kappa } \\
\beta \text {, mTOR inhibitor }\end{array}$ & {$[303]$} & Mild nausea or diarrhea, iron deficiency & {$[304]$} \\
\hline MithramycinA & $\begin{array}{l}\text { Antineoplastic antibiotic, RNA } \\
\text { synthesis inhibitor }\end{array}$ & {$[305]$} & $\begin{array}{l}\text { Changes in lung function, reduction in the } \\
\text { number of white blood cells and platelets } \\
\text { and bleeding }\end{array}$ & {$[306]$} \\
\hline NSC 14778 & Non-covalent inhibitor with a new scaffold & {$[307]$} & N/A & - \\
\hline Nanaomycin A & Antifungal antibiotic, DNMT3B inhibitor & [308] & N/A & - \\
\hline
\end{tabular}


T3 might have beneficial effects on progeria symptoms, as well as aging [81, 82].

\section{PI3K pathway inhibitors}

In T98G cells stimulated with the growth factor PDGF, inhibition with a small molecule inhibitor of PI3K (LY294002), which prevents Akt phosphorylation, has been found to decrease expression of human $L M N A$ mRNA induced by the PDGF-BB. This demonstrates that LMNA may be regulated through the phosphatidylinositol 3-kinase PI3K pathway [83]. It has been suggested that aberrant phosphorylation of Ser458 of Lamin A by Akt1 contributes to striated muscle laminopathies caused by LMNA mutation [84]. Therefore, inhibition of Akt1 by LY294002 might be beneficial to this and similar laminopathies.

\section{Epigenetics marks reversal}

Reversal of epigenetic marks may represent a novel antiaging target. In an animal model of progeria, Zmpste24deficient mice show hypermethylation and transcriptional silencing of rDNA genes. This effect is reversible through treatment with methyltransferase inhibitors [85]. Therefore, methyltransferase inhibitors (Table 5) could prevent HGPS symptoms. In the same animal model it has been noted that a delayed DNA damage response is a result of histone $\mathrm{H} 4$ acetylation defect. Reversal of this defect by supplying the histone deacetylase inhibitor sodium butyrate in drinking water ameliorated aging-associated effects, and extended the lifespan in the animal model. In addition to accumulation of progerin, aged mice show hypoacetylation of the histone H4K16 [86]. Therefore, treatment with methyltransferase inhibitors (Table 5) and histone deacetylase inhibitors could potentially reduce HGPS symptoms. However, such interventions could give rise to significant side effects and would have to be carefully evaluated and refined before transfer to the clinical setting.

Targeting posttranslational modification: farnesylation inhibitors

Ionafarnib (SCH-66336), a farnesyltransferase inhibitor, has been shown to inhibit prelamin A farnesylation in buccal mucosa cells [87]. Other studies demonstrate that inhibitors of farnesyltransferase (FTIs) ameliorate the phenotype of transgenic G608G LMNA mice [88]. This model is characterized by extensive and progressive loss of vascular smooth muscle cells (VSMCs) of large arterial media [89], similar to effects observed in human HGPS patients [90, 91]. FTIs have also been shown to improve survival and bone integrity in LMNA HG/+ $[92,93]$ and in ZMPSTE24-/- mouse models [94]. The compound FTI-277 may completely restore localization of nuclear antigens in HGPS fibroblasts [59]. The combination of statins and aminobisphosphonates has been shown to inhibit the production of farnesylation and geranylgeranylation modifications of prelamin $\mathrm{A}$ and progerin in Face-1/Zmpste24-defective mice, decreasing senescence-like symptoms and increasing the lifespan of affected mice $[95,96]$. Unfortunately, the FTI treatment has harmful side effects such as centrosome separation and bipolar spindle formation defects, nuclear dysmorphy, and cytotoxicity [97]. In addition, mice, expressing nonfarnesylated progerin variants $(\mathrm{LMNA}(\mathrm{nHG} /+))$, still reveal progeria-like phenotypes, which are not ameliorated by FTI [98].

\section{Directly and selectively targeting mutant Lamin $A$}

Targeting mutant Lamin A RNA: antisense oligonucleotides, RNAi, miRNA, and siRNA

Inhibition of the LMNA miss-spliced site reverses senescenceassociated defects in cell nuclei [20]. Fong et al. demonstrated the effectiveness of antisense oligonucleotide technology and identified an antisense oligonucleotide which is complementary to a site in exon 11 at a $5^{\prime}$ position relative to the alternate splice site in LMNA transcripts. This may be used to decrease alternative splicing in HGPS fibroblasts and moderately reduce progerin levels [99]. Splicing-based therapeutical approaches have been examined using a genetically modified mouse strain that carries an HGPS mutation. Antisense morpholino-based therapy has been developed with the aim of preventing pathogenic LMNA splicing, and alleviating the progeroid phenotype [100].

For the past decade, cancer treatments have been developed based on RNA interference (RNAi) - a mechanism that effectively "shuts down" malfunctioning genes with small noncoding RNA molecules from the families of microRNAs. LMNA transcripts have a myriad of microRNAs with which they specifically interact; hence, an RNAi approach offers potential for targeting misspliced LMNA transcripts. In the brain, the Prelamin A transcript is regulated by the brain-specific microRNA miR-9. The tight shutdown of the LMNA transcript observed in the brain using miR-9 may explain the lack of central nervous system pathology in mouse models of HGPS [101]. Recently, Weidefield et al. have reported on the generation of a conditional inducible microRNA (RNAi) system for the controlled inactivation of LMNA [102]. There is also evidence of differential expression of miRNAs in control versus LMNA-related laminopathy [103]. A systemic application of siRNA, specifically targeted to tissues of interest may offer promising potential in future therapeutic applications. 
Targeting mutant protein accumulation: chemical protein binding

A proteomics approach using matrix-assisted laserdesorption-ionization time of flight (MALDI-TOF) MS [104] has identified that lamin A belongs to a family of poly (ADP-ribose) binding proteins. Nuclear lamin A was found to be covalently bound to acetaminophen (APAP), and also appears to become phosphorylated upon arylation. Lamin A may be associated with disruption of nuclear membrane organization, which may be triggered by the translocation of the 55- to 58-kDa APAP-protein adduct, leading to cell death [105]. Direct lamin A/chemical binding may be explored by designing a molecular sponge that sequesters mutant lamin A, i.e., progerin from the cell.

Gene therapy: nanotherapy, viral vectors, protocells, and targeting progerin for autophagy

Several gene therapies and nanotherapies targeting cellular proteins are currently in development. One such approach to the aggregation of misfolded proteins has been applied in the case of a Hungtinton's neurodegeneration (HD) mouse model (HDR6/1) by targeting proteins for autophagic proteosomal degradation using intrabodies. This may represent an effective strategy if modified for clearance of progerin instead [106]. Genome customization and targeted gene modification of Lamin A mutant alleles using gene-specific engineered nucleases such as zinc finger nucleases or transcription activator-like effector nucleases (TALENs, Cellectis) represents another possible approach [107].

The assortment of gene delivery vehicles for gene therapy products are expanding, and include lentivirals and adeno-associated viral vectors [108] in addition to nonviral gene delivery systems such as lipoplexes, polyplexes, inorganic nanoparticles, quantum dots, and protocells [109-111]. However, despite significant progress in recent years, limitations still persist in refining this technology for use in the clinical setting both in terms of patient safety and efficacy.

\section{Future directions}

The role of lamin A is to maintain nuclear structure and integrity and in doing so, contribute to the health and survival potential of individuals within a population. The consequences of defectiveness in lamin A structure and function are observed, therefore, to be extremely severe, manifesting at several broad-ranging and essential biological levels, which include cell signaling and gene expression. As such, any intervention successful in ensuring the maintenance of LMNA protein function and/or the reduction of LMNA's downstream effects must work within key parameters at each of these regulatory levels. Our analysis has identified potential targets for therapeutic intervention by addressing both causes and effects of LMNA defectiveness. Our findings provide a framework for targeting LMNA defectiveness directly at the genetic level and further downstream by targeting signaling events and other processes which give rise to cellular insult and ultimately disease.

By means of a computational analysis of multiple biological pathways, we have identified a number of plausible therapeutic targets, and outlined 12 possible interventions for regulating defective LMNA expression and protein accumulation (see Fig. 2). These are: (1) IGF-1 and GH balance restorers, (2) Notch signaling inhibitors, (3) reactive oxygen species scavengers, (4) telomerase activators, (5) Rb inhibitors, (6) apoptosis inhibitors, (7) translation- and autophagy-activator inhibitors, (8) cAMP activators, (9) thyroid hormone supplementation, (10) PI3K pathway inhibitors, (11) epigenetics marks reversal, and (12) farnesylation inhibitors. While these targets are highly specific in many cases, collectively they are wide ranging and cover the biological complexities that characterize LMNA-related diseases and the levels at which they manifest. Furthermore, we have presented a comprehensive list of compounds known to act on specific targets within these biologic pathways. A combinatory approach may be applied using this data to develop a therapy or therapies consisting of a combination of several key compounds, potentially including: farnesylation inhibitors, autophagy activators, apoptosis inhibitors, and telomerase activators.

Extensive in vitro studies characterizing the effectiveness of these compounds are required before moving towards translation into clinical practice. In particular, the effects of these compounds on progerin accumulation is of particular interest and may be used as a measure of treatment efficacy; in part, it could also be used in the validation of such interventions. However, there are several outstanding questions that must be answered in order to validate the effectiveness of such interventions and the mechanisms by which they provide benefit. Outstanding questions in this field concern the molecular mechanisms by which laminopathies and related diseases manifest and the processes in which progerin alters cellular phenotype and biological age.

In addition to identifying molecular targets, the approach outlined here has also focused on identifying molecules that can carry out specific functions. The use of small molecules to activate/inhibit the signal transduction pathways that regulate LMNA expression itself represents one such approach. In this manner, lamin $\mathrm{A}$ in addition to progerin may be down-regulated to levels that effectively influence cellular homeostasis to a point at which cells are healthy, producing lower levels of both laminA and progerin, and therefore potentially lowering the rate at which aging occurs. There 
are many small molecules/drugs presently available and approved by the FDA (albeit for other purposes), which may be used to target signaling pathways in this way (Tables 1, 2, 3, 4 and 5). However, as part of this strategy, it will be necessary to further characterize the significance of the ratio of progerin/laminA in disease pathogenesis. A long-term goal may be to develop a method of directly targeting of the underlying causes of progeria and related conditions. This requires a technological platform that targets and discriminates progerin and other products of disease-causing Lamin A alleles, from functional lamin A. There are potential tools available at present that may be tailored further for this purpose. In particular, it is feasible that progerin may be specifically targeted at the mRNA level using RNAi tools. In order to target toxicity associated with mutant protein accumulation, designer proteases may also be developed to specifically degrade the mutant protein. In addition, the use of intrabodies to bind and target progerin for autophagy also represents a potential means for achieving these aims. Delivery mechanisms will also be key to any such therapeutic intervention, and the use of viral vectors and nanotherapeutic delivery approaches hold much promise as they are developed and refined into the future. Antisense morpholino-based gene therapy also holds much promise. By directing this technique to prevent pathogenic LMNA splicing, Osorio et al. have achieved reductions in progerin accumulation and associated nuclear defects, amelioration of progeroid phenotypes, and an extension of lifespan [100]. While the gene therapy approach requires further refinement, this technology clearly represents the most likely means of ensuring correct splicing and localization of defective LMNA. Drugs which enhance autophagic mechanisms to achieve reductions in progerin accumulation also show considerable potential; however, safer analogues are required [112]. Another approach may be the use of small molecules to ameliorate the effects of progerin accumulation, such as those listed in this review. However promising, further evaluation of the potential impact of these molecules on the disease phenotype are required prior to application in the clinical setting, either individually or in combination with other therapies. Currently, a number of clinical trials are underway to examine the potential therapeutic effects of using statins, FTIs, and bisphosphonate in combination to treat progeria $[113,114]$. Similarly, some of the molecules highlighted in this study may be incorporated into future clinical trials or treatments.

The overall focus of this review has been to identify and highlight different methods that may be used for treating laminopathies, and to a lesser extent, other LMNAassociated human diseases and aging. While some treatments may act to target the downstream effects of progerin accumulation, other treatments may be used to directly alter the ratio of progerin/wild-type protein. Indeed, the most
Fig. 2 Presented are steps of the gene expression cycle of Lamin A/C that can be targeted for additional regulation: transcription, splicing, translation, posttranslational modification, and degradation via autophagy. See text for a description for strategies for targeting each of these steps with already-available drugs to minimize the deleterious effects of altered Lamin A expression in laminopathies, and possibly aging. Gene names and symbols are the same as those listed in Fig. 1. Red dots represent farnesylation residues on lamin $\mathrm{A} /$ progerin

effective method of treating laminopathies would be to target and counteract progerin accumulation directly. However, the targeting of $L M N A$ expression in general also holds potential for treating patients given that disease severity may be determined in part by the ratio of progerin to mature lamin A. In order for future treatments to significantly alter the ratio of wild-type/mutant protein in favor of cellular health and longevity, mechanisms that achieve increments comparable to wild-type protein levels may be required in addition to also reducing progerin levels. Modulating the expression of Lamin A may also be effecive for the treatment to other human diseases associated with $L M N A$, given that differential allele-specific expression has been identified at the LMNA locus [40]. For example, alleles represented at relatively high frequencies in human populations have been associated with a number of relatively common human diseases (e.g., rs4641). In this respect, development of methods of increasing expression of wild-type LMNA may offer a means of treating both laminopathies and other human diseases association with genetic variation at this locus.

The consequences to intervening to alter the ratio of Progerin/LMNA expression are likely to be significantly influenced by a host of factors, including underlying differences in cell-type and tissues to which treatment is directed. In particular, $L M N A$ expression is known to be developmentally regulated, being expressed in differentiated cells while being absent from early embryonic stem cell compartments and at low to negligible levels in hematopoietic systems [115-117]. This points to the importance of LMNA expression in the maintenance of the differentiated cell state [115]. In HGPS, it has been suggested that stem-cell-driven tissue regeneration may be reduced and tissue-specific differences in apoptosis or regenerative potential may give rise to the tissue-specific segmental aging pattern [118]. Any treatment developed to therapeutically alter the Progerin/LMNA ratio should therefore consider the potential for distinctive role(s) for Lamin A in different tissue compartments.

\section{Conclusions}

In conclusion, our analysis describes a range of potential therapeutic approaches that may be used to modulate the expression and accumulation of defective lamin A and/or modify its downstream effects in laminopathies and age- 


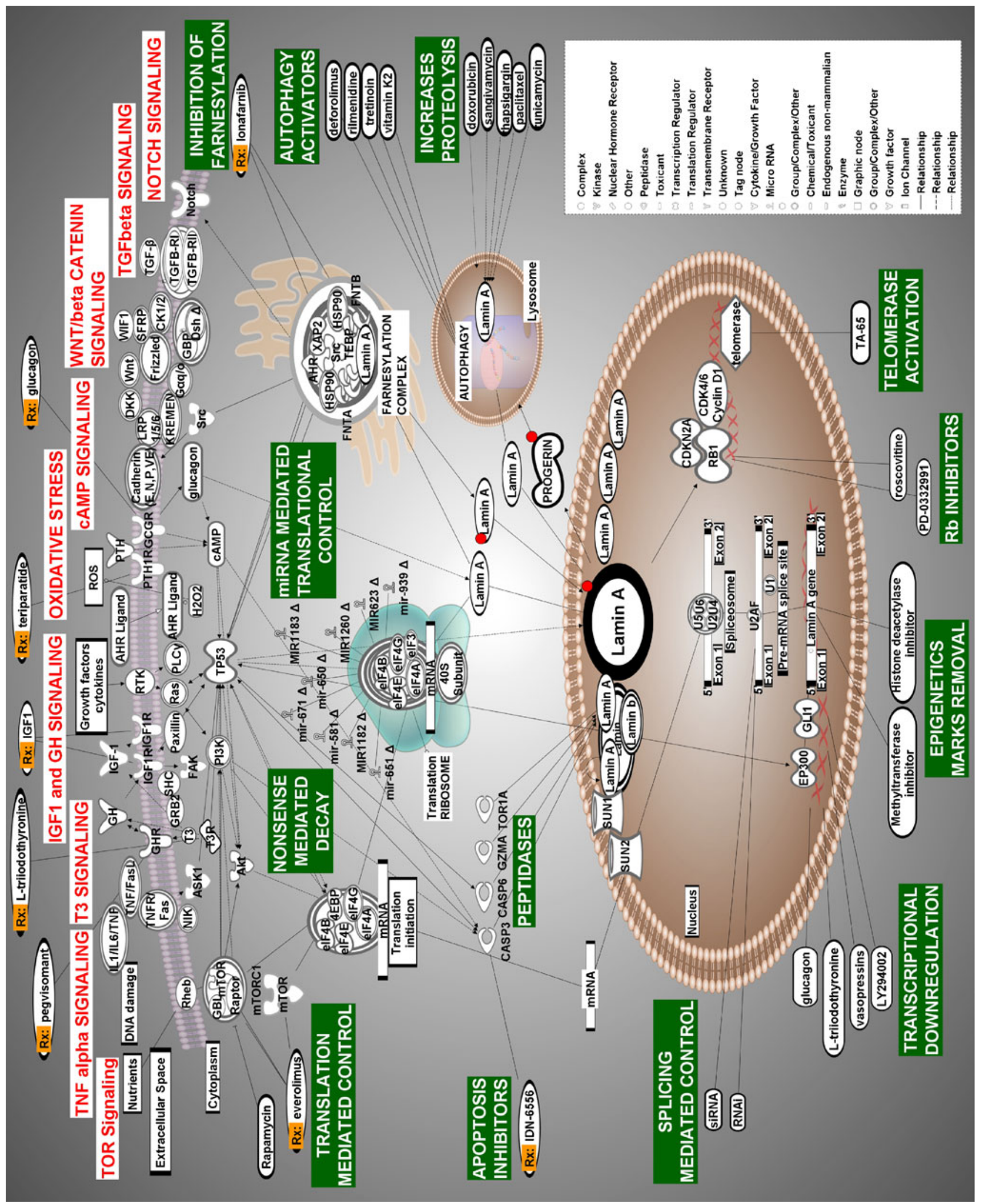


related diseases. However, careful evaluations of these approaches and the potential side effects of drug treatments discussed here are required before consideration as therapeutic treatments in a clinical setting.

Acknowledgments The authors would like to acknowledge Dr. Annalise M. Nawrocki for comments on an earlier version of this manuscript. Generous support for this work was provided by the Retina Macular Degeneration Fund, an AF-IDA grant from UCD and NIH 1R01EY021024-01A2 to ZSM. The work is supported by Grant of Presidium of RAS "Structure-functional organization of chromosomes in cell cycle (12-C-4-1007)" to AAM.

Open Access This article is distributed under the terms of the Creative Commons Attribution License which permits any use, distribution, and reproduction in any medium, provided the original author(s) and the source are credited.

\section{References}

1. Dechat T, Adam SA, Taimen P, Shimi T, Goldman RD (2010) Nuclear lamins. Cold Spring Harb Perspect Biol 2:a000547

2. Dechat T, Gesson K, Foisner R (2010) Lamina-independent lamins in the nuclear interior serve important functions. Cold Spring Harb Symp Quant Biol 75:533-543

3. Silva L, Cliffe A, Chang L, Knipe DM (2008) Role for A-type lamins in herpesviral DNA targeting and heterochromatin modulation. PLoS Pathog 4:e1000071

4. Peter A, Stick R (2012) Evolution of the lamin protein family: what introns can tell. Nucleus 3(1):44-59

5. Lutz RJ, Trujillo MA, Denham KS, Wenger L, Sinensky M (1992) Nucleoplasmic localization of prelamin A: implications for prenylation-dependent lamin A assembly into the nuclear lamina. Proc Natl Acad Sci U S A 89:3000-3004

6. Pendas AM, Zhou Z, Cadinanos J, Freije JM, Wang J, Hultenby $\mathrm{K}$, Astudillo A, Wernerson A, Rodriguez F, Tryggvason $\mathrm{K}$ et al (2002) Defective prelamin A processing and muscular and adipocyte alterations in Zmpste24 metalloproteinase-deficient mice. Nat Genet 31:94-99

7. Columbaro M, Mattioli E, Schena E, Capanni C, Cenni V, Levy N, Navarro CL, Del Coco R, Squarzoni S, Camozzi D et al (2010) Prelamin A processing and functional effects in restrictive dermopathy. Cell Cycle 9:4766-4768

8. Reddy S, Comai L (2012) Lamin A, farnesylation and aging. Exp Cell Res 318:1-7

9. Cao K, Blair CD, Faddah DA, Kieckhaefer JE, Olive M, Erdos MR, Nabel EG, Collins FS (2011) Progerin and telomere dysfunction collaborate to trigger cellular senescence in normal human fibroblasts. J Clin Invest 121:2833-2844

10. Pollex RL, Hegele RA (2004) Hutchinson-Gilford progeria syndrome. Clin Genet 66:375-381

11. Eriksson M, Brown WT, Gordon LB, Glynn MW, Singer J, Scott L, Erdos MR, Robbins CM, Moses TY, Berglund P et al (2003) Recurrent de novo point mutations in lamin A cause HutchinsonGilford progeria syndrome. Nature 423:293-298

12. Lopez-Mejia IC, Vautrot V, De Toledo M, Behm-Ansmant I, Bourgeois CF, Navarro CL, Osorio FG, Freije JM, Stevenin J, De Sandre-Giovannoli A et al (2011) A conserved splicing mechanism of the LMNA gene controls premature aging. Hum Mol Genet 20:4540-4555

13. Moulson CL, Fong LG, Gardner JM, Farber EA, Go G, Passariello A, Grange DK, Young SG, Miner JH (2007)
Increased progerin expression associated with unusual LMNA mutations causes severe progeroid syndromes. Hum Mutat 28:882-889

14. Reunert J, Wentzell R, Walter M, Jakubiczka S, Zenker M, Brune T, Rust S, Marquardt T (2012) Neonatal progeria: increased ratio of progerin to lamin A leads to progeria of the newborn. Eur $\mathrm{J}$ Hum Genet 20(9):933-937

15. Kelley JB, Datta S, Snow CJ, Chatterjee M, Ni L, Spencer A, Yang CS, Cubenas-Potts C, Matunis MJ, Paschal BM (2011) The defective nuclear lamina in Hutchinson-gilford progeria syndrome disrupts the nucleocytoplasmic Ran gradient and inhibits nuclear localization of Ubc9. Mol Cell Biol 31:3378-3395

16. Goldman RD, Shumaker DK, Erdos MR, Eriksson M, Goldman AE, Gordon LB, Gruenbaum Y, Khuon S, Mendez M, Varga R et al (2004) Accumulation of mutant lamin A causes progressive changes in nuclear architecture in Hutchinson-Gilford progeria syndrome. Proc Natl Acad Sci U S A 101:8963-8968

17. Burtner CR, Kennedy BK (2010) Progeria syndromes and ageing: what is the connection? Nat Rev Mol Cell Biol 11:567-578

18. de Magalhaes JP, Budovsky A, Lehmann G, Costa J, Li Y, Fraifeld V, Church GM (2009) The Human Ageing Genomic Resources: online databases and tools for biogerontologists. Aging Cell 8:65-72

19. de Magalhaes JP, Wuttke D, Wood SH, Plank M, Vora C (2012) Genome-environment interactions that modulate aging: powerful targets for drug discovery. Pharmacol Rev 64:88-101

20. Scaffidi P, Misteli T (2006) Lamin A-dependent nuclear defects in human aging. Science 312:1059-1063

21. Ragnauth CD, Warren DT, Liu Y, McNair R, Tajsic T, Figg N, Shroff R, Skepper J, Shanahan CM (2010) Prelamin A acts to accelerate smooth muscle cell senescence and is a novel biomarker of human vascular aging. Circulation 121:2200-2210

22. Candelario J, Borrego S, Reddy S, Comai L (2011) Accumulation of distinct prelamin A variants in human diploid fibroblasts differentially affects cell homeostasis. Exp Cell Res 317:319-329

23. Hegele RA, Huff MW, Young TK (2001) Common genomic variation in LMNA modulates indexes of obesity in Inuit. J Clin Endocrinol Metab 86:2747-2751

24. Weyer C, Wolford JK, Hanson RL, Foley JE, Tataranni PA, Bogardus C, Pratley RE (2001) Subcutaneous abdominal adipocyte size, a predictor of type 2 diabetes, is linked to chromosome 1q21-q23 and is associated with a common polymorphism in LMNA in Pima Indians. Mol Genet Metab 72:231-238

25. Wolford JK, Hanson RL, Bogardus C, Prochazka M (2001) Analysis of the lamin $\mathrm{A} / \mathrm{C}$ gene as a candidate for type II diabetes susceptibility in Pima Indians. Diabetologia 44:779-782

26. Murase Y, Yagi K, Katsuda Y, Asano A, Koizumi J, Mabuchi H (2002) An LMNA variant is associated with dyslipidemia and insulin resistance in the Japanese. Metabolism 51:1017-1021

27. Steinle NI, Kazlauskaite R, Imumorin IG, Hsueh WC, Pollin TI, O'Connell JR, Mitchell BD, Shuldiner AR (2004) Variation in the lamin A/C gene: associations with metabolic syndrome. Arterioscler Thromb Vasc Biol 24:1708-1713

28. Liang H, Murase Y, Katuta Y, Asano A, Kobayashi J, Mabuchi H (2005) Association of LMNA 1908C/T polymorphism with cerebral vascular disease and diabetic nephropathy in Japanese men with type 2 diabetes. Clin Endocrinol (Oxf) 63:317-322

29. Owen KR, Groves CJ, Hanson RL, Knowler WC, Shuldiner AR, Elbein SC, Mitchell BD, Froguel P, Ng MC, Chan JC et al (2007) Common variation in the LMNA gene (encoding lamin $\mathrm{A} / \mathrm{C}$ ) and type 2 diabetes: association analyses in 9,518 subjects. Diabetes 56:879-883

30. Mesa JL, Loos RJ, Franks PW, Ong KK, Luan J, O'Rahilly S, Wareham NJ, Barroso I (2007) Lamin A/C polymorphisms, type 2 diabetes, and the metabolic syndrome: case-control and quantitative trait studies. Diabetes 56:884-889 
31. Wegner L, Andersen G, Sparso T, Grarup N, Glumer C, Borch-Johnsen $\mathrm{K}$, Jorgensen $\mathrm{T}$, Hansen $\mathrm{T}$, Pedersen $\mathrm{O}$ (2007) Common variation in LMNA increases susceptibility to type 2 diabetes and associates with elevated fasting glycemia and estimates of body fat and height in the general population: studies of 7,495 Danish whites. Diabetes 56:694698

32. Duesing K, Charpentier G, Marre M, Tichet J, Hercberg S, Froguel P, Gibson F (2008) Evaluating the association of common LMNA variants with type 2 diabetes and quantitative metabolic phenotypes in French Europids. Diabetologia $51: 76-81$

33. Akasaka H, Katsuya T, Saitoh S, Sugimoto K, Ohnishi H, Congrains A, Ohnishi M, Ohishi M, Rakugi H, Ogihara T et al (2009) A promoter polymorphism of lamin A/C gene is an independent genetic predisposition to arterial stiffness in a Japanese general population (the Tanno and Sobetsu study). J Atheroscler Thromb 16:404-409

34. Urbanek M, Nampiaparampil G, D'Souza J, Sefton E, Ackerman C, Legro RS, Dunaif A (2009) The role of genetic variation in the lamin a/c gene in the etiology of polycystic ovary syndrome. $J$ Clin Endocrinol Metab 94:2665-2669

35. Fontaine-Bisson B, Alessi MC, Saut N, Fumeron F, Marre M, Dutour A, Badens C, Levy N, Tichet J, Juhan-Vague I et al (2010) Polymorphisms of the lamina maturation pathway and their association with the metabolic syndrome: the DESIR prospective study. J Mol Med (Berl) 88:193-201

36. Halaschek-Wiener J, Amirabbasi-Beik M, Monfared N, Pieczyk M, Sailer C, Kollar A, Thomas R, Agalaridis G, Yamada S, Oliveira L et al (2009) Genetic variation in healthy oldest-old. PLoS One 4:e6641

37. Yeh HL, Hou SJ, Yen FC, Hong CJ, Liou YJ, Yang AC, Liu ME, Tsai SJ (2011) Polymorphisms in LMNA and near a SERPINA13 gene are not associated with cognitive performance in Chinese elderly males without dementia. Neurosci Lett 504:32-34

38. Conneely KN, Capell BC, Erdos MR, Sebastiani P, Solovieff N, Swift AJ, Baldwin CT, Budagov T, Barzilai N, Atzmon G et al (2012) Human longevity and common variations in the LMNA gene: a meta-analysis. Aging Cell 11(3):475-481

39. Lin F, Worman HJ (1993) Structural organization of the human gene encoding nuclear lamin A and nuclear lamin C. J Biol Chem 268:16321-16326

40. Rodriguez S, Eriksson M (2011) Low and high expressing alleles of the LMNA gene: implications for laminopathy disease development. PLoS One 6:e25472

41. The International HapMap Consortium (2003) The International HapMap Project. Nature 426:789-796

42. Chen CY, Chi YH, Mutalif RA, Starost MF, Myers TG, Anderson SA, Stewart CL, Jeang KT (2012) Accumulation of the inner nuclear envelope protein sun 1 is pathogenic in progeric and dystrophic laminopathies. Cell 149:565-577

43. Kenyon CJ (2010) The genetics of ageing. Nature 464:504-512

44. Ugalde AP, Marino G, Lopez-Otin C (2010) Rejuvenating somatotropic signaling: a therapeutical opportunity for premature aging? Aging (Albany NY) 2:1017-1022

45. Marino G, Ugalde AP, Fernandez AF, Osorio FG, Fueyo A, Freije JM, Lopez-Otin C (2010) Insulin-like growth factor 1 treatment extends longevity in a mouse model of human premature aging by restoring somatotroph axis function. Proc Natl Acad Sci U S A 107:16268-16273

46. Arnaldez FI, Helman LJ (2012) Targeting the insulin growth factor receptor 1. Hematol Oncol Clin N Am 26:527-542, vii-viii

47. Espada J, Varela I, Flores I, Ugalde AP, Cadinanos J, Pendas AM, Stewart CL, Tryggvason K, Blasco MA, Freije JM et al (2008) Nuclear envelope defects cause stem cell dysfunction in premature-aging mice. J Cell Biol 181:27-35
48. Scaffidi P, Misteli T (2008) Lamin A-dependent misregulation of adult stem cells associated with accelerated ageing. Nat Cell Biol $10: 452-459$

49. Meshorer E, Gruenbaum Y (2008) Gone with the Wnt/Notch: stem cells in laminopathies, progeria, and aging. J Cell Biol 181:9-13

50. Maekawa Y, Minato Y, Ishifune C, Kurihara T, Kitamura A, Kojima H, Yagita H, Sakata-Yanagimoto M, Saito T, Taniuchi I et al (2008) Notch2 integrates signaling by the transcription factors RBP-J and CREB1 to promote T cell cytotoxicity. Nat Immunol 9:1140-1147

51. Kopan R (ed) (2010) Notch signalling, 1st edn. Waltham, Academic Press

52. Richards SA, Muter J, Ritchie P, Lattanzi G, Hutchison CJ (2011) The accumulation of un-repairable DNA damage in laminopathy progeria fibroblasts is caused by ROS generation and is prevented by treatment with $\mathrm{N}$-acetyl cysteine. Hum Mol Genet 20:3997-4004

53. Constantinescu D, Csoka AB, Navara CS, Schatten GP (2010) Defective DSB repair correlates with abnormal nuclear morphology and is improved with FTI treatment in Hutchinson-Gilford progeria syndrome fibroblasts. Exp Cell Res 316:2747-2759

54. Podmore ID, Griffiths HR, Herbert KE, Mistry N, Mistry P, Lunec J (1998) Vitamin C exhibits pro-oxidant properties. Nature 392:559

55. Cai L, Koropatnick J, Cherian MG (2001) Roles of vitamin C in radiation-induced DNA damage in presence and absence of copper. Chem Biol Interact 137:75-88

56. Guidarelli A, De Sanctis R, Cellini B, Fiorani M, Dacha M, Cantoni O (2001) Intracellular ascorbic acid enhances the DNA single-strand breakage and toxicity induced by peroxynitrite in U937 cells. Biochem J 356:509-513

57. Benson EK, Lee SW, Aaronson SA (2010) Role of progerininduced telomere dysfunction in HGPS premature cellular senescence. J Cell Sci 123:2605-2612

58. de Jesus BB, Schneeberger K, Vera E, Tejera A, Harley CB, Blasco MA (2011) The telomerase activator TA-65 elongates short telomeres and increases health span of adult/old mice without increasing cancer incidence. Aging Cell 10:604-621

59. Mehta IS, Bridger JM, Kill IR (2010) Progeria, the nucleolus and farnesyltransferase inhibitors. Biochem Soc Trans 38:287-291

60. Marji J, O'Donoghue SI, McClintock D, Satagopam VP, Schneider R, Ratner D, Worman HJ, Gordon LB, Djabali K (2010) Defective lamin A-Rb signaling in Hutchinson-Gilford progeria syndrome and reversal by farnesyltransferase inhibition. PLoS One 5:e11132

61. Heijink DM, Fehrmann RS, de Vries EG, Koornstra JJ, Oosterhuis D, van der Zee AG, Kleibeuker JH, de Jong S (2011) A bioinformatical and functional approach to identify novel strategies for chemoprevention of colorectal cancer. Oncogene 30:2026-2036

62. Dechat T, Shimi T, Adam SA, Rusinol AE, Andres DA, Spielmann HP, Sinensky MS, Goldman RD (2007) Alterations in mitosis and cell cycle progression caused by a mutant lamin A known to accelerate human aging. Proc Natl Acad Sci U S A 104:4955-4960

63. Bridger JM, Kill IR (2004) Aging of Hutchinson-Gilford progeria syndrome fibroblasts is characterised by hyperproliferation and increased apoptosis. Exp Gerontol 39:717-724

64. Cao K, Graziotto JJ, Blair CD, Mazzulli JR, Erdos MR, Krainc D, Collins FS (2011) Rapamycin reverses cellular phenotypes and enhances mutant protein clearance in Hutchinson-Gilford progeria syndrome cells. Sci Transl Med 3:89ra58

65. Blagosklonny MV (2011) Progeria, rapamycin and normal aging: recent breakthrough. Aging (Albany NY) 3:685-691

66. Cenni V, Capanni C, Columbaro M, Ortolani M, D'Apice MR, Novelli G, Fini M, Marmiroli S, Scarano E, Maraldi NM et al 
(2011) Autophagic degradation of farnesylated prelamin A as a therapeutic approach to lamin-linked progeria. Eur J Histochem $55: \mathrm{e} 36$

67. Rose C, Menzies FM, Renna M, Acevedo-Arozena A, Corrochano S, Sadiq O, Brown SD, Rubinsztein DC (2010) Rilmenidine attenuates toxicity of polyglutamine expansions in a mouse model of Huntington's disease. Hum Mol Genet 19:2144-2153

68. Rajawat Y, Hilioti Z, Bossis I (2010) Autophagy: a target for retinoic acids. Autophagy 6:1224-1226

69. Rajawat Y, Hilioti Z, Bossis I (2011) Retinoic acid induces autophagosome maturation through redistribution of the cationindependent mannose-6-phosphate receptor. Antioxid Redox Signal 14:2165-2177

70. Kawakita H, Tsuchida A, Miyazawa K, Naito M, Shigoka M, Kyo B, Enomoto M, Wada T, Katsumata K, Ohyashiki K et al (2009) Growth inhibitory effects of vitamin K2 on colon cancer cell lines via different types of cell death including autophagy and apoptosis. Int J Mol Med 23:709-716

71. Stravopodis DJ, Karkoulis PK, Konstantakou EG, Melachroinou $\mathrm{S}$, Lampidonis AD, Anastasiou D, Kachrilas S, Messini-Nikolaki N, Papassideri IS, Aravantinos G et al (2009) Grade-dependent effects on cell cycle progression and apoptosis in response to doxorubicin in human bladder cancer cell lines. Int J Oncol $34: 137-160$

72. Hundeshagen P, Hamacher-Brady A, Eils R, Brady NR (2011) Concurrent detection of autolysosome formation and lysosomal degradation by flow cytometry in a high-content screen for inducers of autophagy. BMC Biol 9:38

73. Lee SA, Jung M (2007) The nucleoside analog sangivamycin induces apoptotic cell death in breast carcinoma MCF7/adriamycin-resistant cells via protein kinase Cdelta and JNK activation. J Biol Chem 282:15271-15283

74. Siman R, Flood DG, Thinakaran G, Neumar RW (2001) Endoplasmic reticulum stress-induced cysteine protease activation in cortical neurons: effect of an Alzheimer's disease-linked presenilin-1 knock-in mutation. J Biol Chem 276:44736-44743

75. Arnt CR, Chiorean MV, Heldebrant MP, Gores GJ, Kaufmann SH (2002) Synthetic Smac/DIABLO peptides enhance the effects of chemotherapeutic agents by binding XIAP and cIAP1 in situ. J Biol Chem 277:44236-44243

76. Gunaratne R, Braucht DW, Rinschen MM, Chou CL, Hoffert JD, Pisitkun T, Knepper MA (2010) Quantitative phosphoproteomic analysis reveals cAMP/vasopressin-dependent signaling pathways in native renal thick ascending limb cells. Proc Natl Acad Sci U S A 107:15653-15658

77. Georgatos SD, Stournaras C, Blobel G (1988) Heterotypic and homotypic associations between the nuclear lamins: sitespecificity and control by phosphorylation. Proc Natl Acad Sci U S A 85:4325-4329

78. Feng X, Jiang Y, Meltzer P, Yen PM (2000) Thyroid hormone regulation of hepatic genes in vivo detected by complementary DNA microarray. Mol Endocrinol 14:947-955

79. Kivuva EC, Parker MJ, Cohen MC, Wagner BE, Sobey G (2008) De Barsy syndrome: a review of the phenotype. Clin Dysmorphol 17:99-107

80. Ioan D, Dumitriu L, Belengeanu V, Bistriceanu M, Maximilian C (1988) Leprechaunism: report of two cases and review. Endocrinologie 26:205-209

81. Suzuki S, Nishio S, Takeda T, Komatsu M (2012) Genderspecific regulation of response to thyroid hormone in aging. Thyroid Res 5:1

82. Faggiano A, Del Prete M, Marciello F, Marotta V, Ramundo V, Colao A (2011) Thyroid diseases in elderly. Minerva Endocrinol $36: 211-231$

83. Tullai JW, Schaffer ME, Mullenbrock S, Kasif S, Cooper GM (2004) Identification of transcription factor binding sites upstream of human genes regulated by the phosphatidylinositol 3-kinase and MEK/ERK signaling pathways. J Biol Chem 279:20167-20177

84. Mitsuhashi H, Hayashi YK, Matsuda C, Noguchi S, Wakatsuki S, Araki T, Nishino I (2010) Specific phosphorylation of Ser458 of A-type lamins in LMNA-associated myopathy patients. J Cell Sci 123:3893-3900

85. Osorio FG, Varela I, Lara E, Puente XS, Espada J, Santoro R, Freije JM, Fraga MF, Lopez-Otin C (2010) Nuclear envelope alterations generate an aging-like epigenetic pattern in mice deficient in Zmpste24 metalloprotease. Aging Cell 9:947-957

86. Krishnan V, Chow MZ, Wang Z, Zhang L, Liu B, Liu X, Zhou Z (2011) Histone H4 lysine 16 hypoacetylation is associated with defective DNA repair and premature senescence in Zmpste24deficient mice. Proc Natl Acad Sci U S A 108:12325-12330

87. Adjei AA, Erlichman C, Davis JN, Cutler DL, Sloan JA, Marks RS, Hanson LJ, Svingen PA, Atherton P, Bishop WR et al (2000) A Phase I trial of the farnesyl transferase inhibitor SCH66336: evidence for biological and clinical activity. Cancer Res 60:18711877

88. Capell BC, Olive M, Erdos MR, Cao K, Faddah DA, Tavarez UL, Conneely KN, Qu X, San H, Ganesh SK et al (2008) A farnesyltransferase inhibitor prevents both the onset and late progression of cardiovascular disease in a progeria mouse model. Proc Natl Acad Sci U S A 105:15902-15907

89. Varga R, Eriksson M, Erdos MR, Olive M, Harten I, Kolodgie F, Capell BC, Cheng J, Faddah D, Perkins S et al (2006) Progressive vascular smooth muscle cell defects in a mouse model of Hutchinson-Gilford progeria syndrome. Proc Natl Acad Sci U S A 103:3250-3255

90. Capell BC, Collins FS, Nabel EG (2007) Mechanisms of cardiovascular disease in accelerated aging syndromes. Circ Res 101:13-26

91. Stehbens WE, Delahunt B, Shozawa T, Gilbert-Barness E (2001) Smooth muscle cell depletion and collagen types in progeric arteries. Cardiovasc Pathol 10:133-136

92. Yang SH, Meta M, Qiao X, Frost D, Bauch J, Coffinier C, Majumdar S, Bergo MO, Young SG, Fong LG (2006) A farnesyltransferase inhibitor improves disease phenotypes in mice with a Hutchinson-Gilford progeria syndrome mutation. J Clin Invest 116:2115-2121

93. Yang SH, Qiao X, Farber E, Chang SY, Fong LG, Young SG (2008) Eliminating the synthesis of mature lamin A reduces disease phenotypes in mice carrying a Hutchinson-Gilford progeria syndrome allele. J Biol Chem 283:7094-7099

94. Fong LG, Frost D, Meta M, Qiao X, Yang SH, Coffinier C, Young SG (2006) A protein farnesyltransferase inhibitor ameliorates disease in a mouse model of progeria. Science 311:16211623

95. Varela I, Pereira S, Ugalde AP, Navarro CL, Suarez MF, Cau P, Cadinanos J, Osorio FG, Foray N, Cobo J et al (2008) Combined treatment with statins and aminobisphosphonates extends longevity in a mouse model of human premature aging. Nat Med 14:767-772

96. Osorio FG, Obaya AJ, Lopez-Otin C, Freije JM (2009) Accelerated ageing: from mechanism to therapy through animal models. Transgenic Res 18:7-15

97. Verstraeten VL, Peckham LA, Olive M, Capell BC, Collins FS, Nabel EG, Young SG, Fong LG, Lammerding J (2011) Protein farnesylation inhibitors cause donut-shaped cell nuclei attributable to a centrosome separation defect. Proc Natl Acad Sci U S A 108:4997-5002

98. Yang SH, Chang SY, Ren S, Wang Y, Andres DA, Spielmann HP, Fong LG, Young SG (2011) Absence of progeria-like disease phenotypes in knock-in mice expressing a non-farnesylated version of progerin. Hum Mol Genet 20:436-444 
99. Fong LG, Vickers TA, Farber EA, Choi C, Yun UJ, Hu Y, Yang SH, Coffinier C, Lee R, Yin L et al (2009) Activating the synthesis of progerin, the mutant prelamin A in HutchinsonGilford progeria syndrome, with antisense oligonucleotides. Hum Mol Genet 18:2462-2471

100. Osorio FG, Navarro CL, Cadinanos J, Lopez-Mejia IC, Quiros PM, Bartoli C, Rivera J, Tazi J, Guzman G, Varela I et al (2011) Splicing-directed therapy in a new mouse model of human accelerated aging. Sci Transl Med 3:106ra107

101. Jung HJ, Coffinier C, Choe Y, Beigneux AP, Davies BS, Yang SH, Barnes RH 2nd, Hong J, Sun T, Pleasure SJ et al (2012) Regulation of prelamin A but not lamin C by miR-9, a brainspecific microRNA. Proc Natl Acad Sci U S A 109:E423-E431

102. Weidenfeld I (2012) Inducible microRNA-mediated knockdown of the endogenous human lamin A/C gene. Methods Mol Biol 815:289-305

103. Sylvius N, Bonne G, Straatman K, Reddy T, Gant TW, Shackleton S (2011) MicroRNA expression profiling in patients with lamin A/C-associated muscular dystrophy. FASEB J 25:3966-3978

104. Gagne JP, Hunter JM, Labrecque B, Chabot B, Poirier GG (2003) A proteomic approach to the identification of heterogeneous nuclear ribonucleoproteins as a new family of poly(ADP-ribose)-binding proteins. Biochem J 371:331-340

105. Pumford NR, Halmes NC (1997) Protein targets of xenobiotic reactive intermediates. Annu Rev Pharmacol Toxicol 37:91-117

106. Butler DC, Messer A (2011) Bifunctional anti-huntingtin proteasome-directed intrabodies mediate efficient degradation of mutant huntingtin exon 1 protein fragments. PLoS One 6: e29199

107. Kiefer JC (2011) Primer and interviews: advances in targeted gene modification. Dev Dyn 240:2688-2696

108. Yanez-Munoz RJ, Balaggan KS, MacNeil A, Howe SJ, Schmidt M, Smith AJ, Buch P, MacLaren RE, Anderson PN, Barker SE et al (2006) Effective gene therapy with nonintegrating lentiviral vectors. Nat Med 12:348-353

109. Tani J, Faustine SJT (2011) Updates on current advances in gene therapy. West Indian Med J 60:188-194

110. Li JM, Wang YY, Zhao MX, Tan CP, Li YQ, Le XY, Ji LN, Mao ZW (2012) Multifunctional QD-based co-delivery of siRNA and doxorubicin to HeLa cells for reversal of multidrug resistance and real-time tracking. Biomaterials 33:2780-2790

111. Ashley CE, Carnes EC, Phillips GK, Padilla D, Durfee PN, Brown PA, Hanna TN, Liu J, Phillips B, Carter MB et al (2011) The targeted delivery of multicomponent cargos to cancer cells by nanoporous particle-supported lipid bilayers. Nat Mater 10:389-397

112. Graziotto JJ, Cao K, Collins FS, Krainc D (2012) Rapamycin activates autophagy in Hutchinson-Gilford progeria syndrome: implications for normal aging and age-dependent neurodegenerative disorders. Autophagy 8:147-151

113. Children's Hospital Boston. Study of zoledronic acid, pravastatin, and lonafarnib for patients with progeria. In: ClinicalTrials.gov [Internet]. Bethesda (MD): National Library of Medicine (US). 2009-ongoing [cited 2012 Oct 05]. Available from: http://clinical trials.gov/ct2/show/NCT00916747; NLM Identifier: NCT00916747.

114. Assistance Publique Hopitaux De Marseille: Treatment of the Hutchinson-Gilford Progeria Syndrome With a Combination of Pravastatin and Zoledronic Acid, In: ClinicalTrials.gov [Internet]. Bethesda (MD): National Library of Medicine (US). 2009-ongoing [cited 2012 Oct 05]. Available from: http://clinicaltrials.gov/ ct2/show/NCT00731016; NLM Identifier: NCT00731016.

115. Constantinescu D, Gray HL, Sammak PJ, Schatten GP, Csoka AB (2006) Lamin A/C expression is a marker of mouse and human embryonic stem cell differentiation. Stem Cells 24:177-185

116. Rober RA, Sauter H, Weber K, Osborn M (1990) Cells of the cellular immune and hemopoietic system of the mouse lack lamins A/C: distinction versus other somatic cells. J Cell Sci 95 (Pt 4):587-598

117. Rober RA, Weber K, Osborn M (1989) Differential timing of nuclear lamin $\mathrm{A} / \mathrm{C}$ expression in the various organs of the mouse embryo and the young animal: a developmental study. Development 105:365-378

118. Halaschek-Wiener J, Brooks-Wilson A (2007) Progeria of stem cells: stem cell exhaustion in Hutchinson-Gilford progeria syndrome. J Gerontol A Biol Sci Med Sci 62:3-8

119. Wu G, Lyapina S, Das I, Li J, Gurney M, Pauley A, Chui I, Deshaies RJ, Kitajewski J (2001) SEL-10 is an inhibitor of notch signaling that targets notch for ubiquitin-mediated protein degradation. Mol Cell Biol 21:7403-7415

120. Zhang CP, Yang JL, Zhang J, Li L, Huang L, Ji SY, Hu ZY, Gao F, Liu YX (2011) Notch signaling is involved in ovarian follicle development by regulating granulosa cell proliferation. Endocrinology 152:2437-2447

121. Metacore from GeneGo Inc., A Thompson Reuters company, 2012 (http://www.genego.com)

122. Shih IM, Wang TL (2007) Notch signaling, gamma-secretase inhibitors, and cancer therapy. Cancer Res 67:1879-1882

123. Chernoff AE, Granowitz EV, Shapiro L, Vannier E, Lonnemann G, Angel JB, Kennedy JS, Rabson AR, Wolff SM, Dinarello CA (1995) A randomized, controlled trial of IL-10 in humans. Inhibition of inflammatory cytokine production and immune responses. J Immunol 154:5492-5499

124. Tomita T, Iwatsubo T (2004) The inhibition of gamma-secretase as a therapeutic approach to Alzheimer's disease. Drug News Perspect 17:321-325

125. Bekaii-Saab TS, Mortazavi A, Hicks LG, Zalupski M, Pelley RJ, Chan KK, Kraut EH (2006) A phase II study of chloroquinoxaline sulfonamide (CQS) in patients with metastatic colorectal carcinoma (MCRC). Investig New Drugs 24:343-346

126. Wanka J, Jones LI, Wood PH, Dixon AS (1964) Indomethacin in rheumatic diseases. a controlled clinical trial. Ann Rheum Dis 23:218-225

127. Takebe N, Warren RQ, Ivy SP (2011) Breast cancer growth and metastasis: interplay between cancer stem cells, embryonic signaling pathways and epithelial-to-mesenchymal transition. Breast Cancer Res 13:211

128. Scherr DS, Pitts WR Jr (2003) The nonsteroidal effects of diethylstilbestrol: the rationale for androgen deprivation therapy without estrogen deprivation in the treatment of prostate cancer. J Urol 170:1703-1708

129. Huynh C, Poliseno L, Segura MF, Medicherla R, Haimovic A, Menendez S, Shang S, Pavlick A, Shao Y, Darvishian F et al (2011) The novel gamma secretase inhibitor RO4929097 reduces the tumor initiating potential of melanoma. PLoS One 6:e25264

130. Yamada J, Yoshimura S, Yamakawa H, Sawada M, Nakagawa M, Hara S, Kaku Y, Iwama T, Naganawa T, Banno Y et al (2003) Cell permeable ROS scavengers, Tiron and Tempol, rescue PC12 cell death caused by pyrogallol or hypoxia/reoxygenation. Neurosci Res 45:1-8

131. Han YH, Park WH (2009) Tiron, a ROS scavenger, protects human lung cancer Calu- 6 cells against antimycin A-induced cell death. Oncol Rep 21:253-261

132. Stanley JL, Andersson IJ, Hirt CJ, Moore L, Dilworth MR, Chade AR, Sibley CP, Davidge ST, Baker PN (2012) Effect of the antioxidant tempol on fetal growth in a mouse model of fetal growth restriction. Biol Reprod

133. Lee S, Pagoria D, Raigrodski A, Geurtsen W (2007) Effects of combinations of ROS scavengers on oxidative DNA damage caused by visible-light-activated camphorquinone/ $N, N$-dimethylp-toluidine. J Biomed Mater Res B Appl Biomater 83:391-399

134. Stey C, Steurer J, Bachmann S, Medici TC, Tramer MR (2000) The effect of oral $\mathrm{N}$-acetylcysteine in chronic bronchitis: a quantitative systematic review. Eur Respir J 16:253-262 
135. Anonymous (1974) Toxicological evaluation of some food additives including anticaking agents, antimicrobials, antioxidants, emulsifiers and thickening agents. FAO Nutr Meet Rep Ser: 1-520

136. Jialal I, Grundy SM (1992) Effect of dietary supplementation with alpha-tocopherol on the oxidative modification of low density lipoprotein. J Lipid Res 33:899-906

137. Chan AS, Cheung MC, Law SC, Chan JH (2004) Phase II study of alpha-tocopherol in improving the cognitive function of patients with temporal lobe radionecrosis. Cancer 100:398-404

138. Fuks B, Talaga P, Huart C, Henichart JP, Bertrand K, Grimee R, Lorent $\mathrm{G}$ (2005) In vitro properties of 5-(benzylsulfonyl)-4-bromo-2-methyl-3(2H)-pyridazinone: a novel permeability transition pore inhibitor. Eur J Pharmacol 519:24-30

139. Grohm J, Plesnila N, Culmsee C (2010) Bid mediates fission, membrane permeabilization and peri-nuclear accumulation of mitochondria as a prerequisite for oxidative neuronal cell death. Brain Behav Immun 24:831-838

140. Becattini B, Sareth S, Zhai D, Crowell KJ, Leone M, Reed JC, Pellecchia M (2004) Targeting apoptosis via chemical design: inhibition of bid-induced cell death by small organic molecules. Chem Biol 11:1107-1117

141. Wei MC, Lindsten T, Mootha VK, Weiler S, Gross A, Ashiya M, Thompson CB, Korsmeyer SJ (2000) tBID, a membrane-targeted death ligand, oligomerizes BAK to release cytochrome c. Genes Dev 14:2060-2071

142. Kimura H, Sato Y, Tajima Y, Suzuki H, Yukitake H, Imaeda T, Kajino M, Oki H, Takizawa M, Tanida S (2010) BTZO-1, a cardioprotective agent, reveals that macrophage migration inhibitory factor regulates ARE-mediated gene expression. Chem Biol $17: 1282-1294$

143. Furlong IJ, Lopez Mediavilla C, Ascaso R, Lopez Rivas A, Collins MK (1998) Induction of apoptosis by valinomycin: mitochondrial permeability transition causes intracellular acidification. Cell Death Differ 5:214-221

144. Mastrangelo AJ, Zou S, Hardwick JM, Betenbaugh MJ (1999) Antiapoptosis chemicals prolong productive lifetimes of mammalian cells upon Sindbis virus vector infection. Biotechnol Bioeng 65:298-305

145. Dumont A, Hehner SP, Hofmann TG, Ueffing M, Droge W, Schmitz ML (1999) Hydrogen peroxide-induced apoptosis is CD95-independent, requires the release of mitochondria-derived reactive oxygen species and the activation of NF-kappa $\beta$. Oncogene 18:747-757

146. Gross A, McDonnell JM, Korsmeyer SJ (1999) BCL-2 family members and the mitochondria in apoptosis. Genes Dev 13:1899-1911

147. Scarlett JL, Sheard PW, Hughes G, Ledgerwood EC, Ku HH, Murphy MP (2000) Changes in mitochondrial membrane potential during staurosporine-induced apoptosis in Jurkat cells. FEBS Lett 475:267-272

148. Pastorino JG, Hoek JB (2000) Ethanol potentiates tumor necrosis factor-alpha cytotoxicity in hepatoma cells and primary rat hepatocytes by promoting induction of the mitochondrial permeability transition. Hepatology 31:1141-1152

149. Damico R, Simms T, Kim BS, Tekeste Z, Amankwan H, Damarla M, Hassoun PM (2011) p53 mediates cigarette smoke-induced apoptosis of pulmonary endothelial cells: inhibitory effects of macrophage migration inhibitor factor. Am J Respir Cell Mol Biol 44:323-332

150. Zhao CQ, Zhang YH, Jiang SD, Jiang LS, Dai LY (2010) Both endoplasmic reticulum and mitochondria are involved in disc cell apoptosis and intervertebral disc degeneration in rats. Age (Dordr) 32:161-177

151. Chu W, Zhang J, Zeng C, Rothfuss J, Tu Z, Chu Y, Reichert DE, Welch MJ, Mach RH (2005) N-benzylisatin sulfonamide analogues as potent caspase-3 inhibitors: synthesis, in vitro activity, and molecular modeling studies. J Med Chem 48:76377647

152. Lee D, Long SA, Murray JH, Adams JL, Nuttall ME, Nadeau DP, Kikly K, Winkler JD, Sung CM, Ryan MD et al (2001) Potent and selective nonpeptide inhibitors of caspases 3 and 7. J Med Chem 44:2015-2026

153. Hoffstrom BG, Kaplan A, Letso R, Schmid RS, Turmel GJ, Lo DC, Stockwell BR (2010) Inhibitors of protein disulfide isomerase suppress apoptosis induced by misfolded proteins. Nat Chem Biol 6:900-906

154. Cherqui S (2012) Cysteamine therapy: a treatment for cystinosis, not a cure. Kidney Int 81:127-129

155. Tsilou ET, Thompson D, Lindblad AS, Reed GF, Rubin B, Gahl W, Thoene J, Del Monte M, Schneider JA, Granet DB et al (2003) A multicentre randomised double masked clinical trial of a new formulation of topical cysteamine for the treatment of corneal cystine crystals in cystinosis. Br J Ophthalmol 87:28-31

156. Komarova EA, Gudkov AV (2000) Suppression of p53: a new approach to overcome side effects of antitumor therapy. Biochemistry (Mosc) 65:41-48

157. Komarov PG, Komarova EA, Kondratov RV, Christov-Tselkov K, Coon JS, Chernov MV, Gudkov AV (1999) A chemical inhibitor of p53 that protects mice from the side effects of cancer therapy. Science 285:1733-1737

158. Strom E, Sathe S, Komarov PG, Chernova OB, Pavlovska I, Shyshynova I, Bosykh DA, Burdelya LG, Macklis RM, Skaliter R et al (2006) Small-molecule inhibitor of p53 binding to mitochondria protects mice from gamma radiation. Nat Chem Biol 2:474-479

159. Elimadi A, Jullien V, Tillement JP, Morin D (2003) S-15176 inhibits mitochondrial permeability transition via a mechanism independent of its antioxidant properties. Eur J Pharmacol 468:93-101

160. McCall M, Toso C, Emamaullee J, Pawlick R, Edgar R, Davis J, Maciver A, Kin T, Arch R, Shapiro AM (2011) The caspase inhibitor IDN-6556 (PF3491390) improves marginal mass engraftment after islet transplantation in mice. Surgery 150:48-55

161. Rao VA, Klein SR, Bonar SJ, Zielonka J, Mizuno N, Dickey JS, Keller PW, Joseph J, Kalyanaraman B, Shacter E (2010) The antioxidant transcription factor $\mathrm{Nrf} 2$ negatively regulates autophagy and growth arrest induced by the anticancer redox agent mitoquinone. J Biol Chem 285:34447-34459

162. Deretic V (2012) Autophagy as an innate immunity paradigm: expanding the scope and repertoire of pattern recognition receptors. Curr Opin Immunol 24:21-31

163. Vieth R, Chan PC, MacFarlane GD (2001) Efficacy and safety of vitamin D3 intake exceeding the lowest observed adverse effect level. Am J Clin Nutr 73:288-294

164. Wang Q, Liang B, Shirwany NA, Zou MH (2011) 2-Deoxy-Dglucose treatment of endothelial cells induces autophagy by reactive oxygen species-mediated activation of the AMP-activated protein kinase. PLoS One 6:e17234

165. Xi H, Kurtoglu M, Liu H, Wangpaichitr M, You M, Liu X, Savaraj N, Lampidis TJ (2011) 2-Deoxy-D-glucose activates autophagy via endoplasmic reticulum stress rather than ATP depletion. Cancer Chemother Pharmacol 67:899-910

166. Bijnsdorp IV, Peters GJ, Temmink OH, Fukushima M, Kruyt FA (2010) Differential activation of cell death and autophagy results in an increased cytotoxic potential for trifluorothymidine compared to 5-fluorouracil in colon cancer cells. Int $\mathrm{J}$ Cancer 126:2457-2468

167. Lin Q, Gao XS, Qiao XY, Chen K, Wang YD, Zhou ZG (2008) Phase II clinical trial of concurrent chemoradiotherapy (cisplatin plus 5-fluorouracil) for esophageal cancer. Ai Zheng 27:10771081 
168. Xia LP, Li LY, Fei XF, Liang ZQ (2010) Autophagy is involved in 6-OHDA-induced dopaminergic cell death. Nan Fang Yi Ke Da Xue Xue Bao 30:2649-2651

169. Ding WX, Ni HM, Gao W, Hou YF, Melan MA, Chen X, Stolz DB, Shao ZM, Yin XM (2007) Differential effects of endoplasmic reticulum stress-induced autophagy on cell survival. J Biol Chem 282:4702-4710

170. Balgi AD, Fonseca BD, Donohue E, Tsang TC, Lajoie P, Proud CG, Nabi IR, Roberge M (2009) Screen for chemical modulators of autophagy reveals novel therapeutic inhibitors of mTORC1 signaling. PLoS One 4:e7124

171. Naccarelli GV, Rinkenberger RL, Dougherty AH, Fitzgerald DM (1989) Adverse effects of amiodarone. Pathogenesis, incidence and management. Med Toxicol Adverse Drug Exp 4:246-253

172. Cheong H, Lindsten T, Thompson CB (2012) Autophagy and ammonia. Autophagy 8(1):122-123

173. Goussetis DJ, Altman JK, Glaser H, McNeer JL, Tallman MS, Platanias LC (2010) Autophagy is a critical mechanism for the induction of the antileukemic effects of arsenic trioxide. J Biol Chem 285:29989-29997

174. Liu SY, Wen CY, Lee YJ, Lee TC (2010) XPC silencing sensitizes glioma cells to arsenic trioxide via increased oxidative damage. Toxicol Sci 116:183-193

175. Berenson JR, Matous J, Swift RA, Mapes R, Morrison B, Yeh HS (2007) A phase I/II study of arsenic trioxide/bortezomib/ascorbic acid combination therapy for the treatment of relapsed or refractory multiple myeloma. Clin Cancer Res 13:1762-1768

176. Din FV, Valanciute A, Houde V, Zibrova D, Green KA, Sakamoto K, Alessi DR, Dunlop MG (2012) Aspirin inhibits mTOR signaling, activates AMP-activated protein kinase, and induces autophagy in colorectal cancer cells. Gastroenterology 142 (7):1504-15.e3

177. Sandler RS, Halabi S, Baron JA, Budinger S, Paskett E, Keresztes R, Petrelli N, Pipas JM, Karp DD, Loprinzi CL et al (2003) A randomized trial of aspirin to prevent colorectal adenomas in patients with previous colorectal cancer. N Engl J Med 348:883-890

178. Zhang Q, Yang YJ, Wang H, Dong QT, Wang TJ, Qian HY, Xu H (2012) Autophagy activationA novel mechanism of atorvastatin to protect mesenchymal stem cells from hypoxia and serum deprivation via AMP-activated protein kinase/mammalian target of rapamycin pathway. Stem Cells Dev 21(8):1321-1332

179. Newman C, Tsai J, Szarek M, Luo D, Gibson E (2006) Comparative safety of atorvastatin $80 \mathrm{mg}$ versus $10 \mathrm{mg}$ derived from analysis of 49 completed trials in 14,236 patients. Am J Cardiol 97:61-67

180. Pattingre S, Bauvy C, Codogno P (2003) Amino acids interfere with the ERK1/2-dependent control of macroautophagy by controlling the activation of Raf- 1 in human colon cancer HT-29 cells. J Biol Chem 278:16667-16674

181. Schenone S, Brullo C, Musumeci F, Radi M, Botta M (2011) ATP-competitive inhibitors of mTOR: an update. Curr Med Chem 18:2995-3014

182. Ariyoshi-Kishino K, Hashimoto K, Amano O, Saitoh J, Kochi M, Sakagami H (2010) Tumor-specific cytotoxicity and type of cell death induced by benzaldehyde. Anticancer Res 30:5069-5076

183. Jia L, Gopinathan G, Sukumar JT, Gribben JG (2012) Blocking autophagy prevents bortezomib-induced NF- $\mathrm{K} \beta$ activation by reducing I-K $\beta \alpha$ degradation in lymphoma cells. PLoS One 7: e32584

184. Komatsu S, Miyazawa K, Moriya S, Takase A, Naito M, Inazu M, Kohno N, Itoh M, Tomoda A (2012) Clarithromycin enhances bortezomib-induced cytotoxicity via endoplasmic reticulum stress-mediated CHOP (GADD153) induction and autophagy in breast cancer cells. Int J Oncol 40:1029-1039
185. Phuphanich S, Supko JG, Carson KA, Grossman SA, Burt Nabors L, Mikkelsen T, Lesser G, Rosenfeld S, Desideri S, Olson JJ (2010) Phase 1 clinical trial of bortezomib in adults with recurrent malignant glioma. J Neurooncol 100:95-103

186. Kim DS, Kim JH, Lee GH, Kim HT, Lim JM, Chae SW, Chae HJ, Kim HR (2010) p38 Mitogen-activated protein kinase is involved in endoplasmic reticulum stress-induced cell death and autophagy in human gingival fibroblasts. Biol Pharm Bull 33:545-549

187. Bae N, Ahn T, Chung S, Oh MS, Ko H, Oh H, Park G, Yang HO (2011) The neuroprotective effect of modified Yeoldahanso-tang via autophagy enhancement in models of Parkinson's disease. J Ethnopharmacol 134:313-322

188. Pfisterer SG, Mauthe M, Codogno P, Proikas-Cezanne T (2011) $\mathrm{Ca} 2+/$ calmodulin-dependent kinase $(\mathrm{CaMK})$ signaling via CaMKI and AMP-activated protein kinase contributes to the regulation of WIPI-1 at the onset of autophagy. Mol Pharmacol 80:1066-1075

189. Yoon JH, Ahn SG, Lee BH, Jung SH, Oh SH (2012) Role of autophagy in chemoresistance: regulation of the ATM-mediated DNA-damage signaling pathway through activation of DNAPKcs and PARP-1. Biochem Pharmacol 83:747-757

190. Goldfrank LR, Hoffman RS (2007) Goldfrank's manual of toxicologic emergencies. McGraw-Hill Medical, New York

191. Kim H, Bernard ME, Flickinger J, Epperly MW, Wang H, Dixon TM, Shields D, Houghton F, Zhang X, Greenberger JS (2011) The autophagy-inducing drug carbamazepine is a radiation protector and mitigator. Int J Radiat Biol 87:1052-1060

192. Xiong N, Jia M, Chen C, Xiong J, Zhang Z, Huang J, Hou L, Yang H, Cao X, Liang Z et al (2011) Potential autophagy enhancers attenuate rotenone-induced toxicity in SH-SY5Y. Neuroscience 199:292-302

193. Dam M, Christiansen J, Kristensen CB, Helles A, Jaegerskou A, Schmiegelow M (1981) Carbamazepine: a clinical biopharmaceutical study. Eur J Clin Pharmacol 20:59-64

194. Pattingre S, Bauvy C, Levade T, Levine B, Codogno P (2009) Ceramide-induced autophagy: to junk or to protect cells? Autophagy 5:558-560

195. Daido S, Kanzawa T, Yamamoto A, Takeuchi H, Kondo Y, Kondo S (2004) Pivotal role of the cell death factor BNIP3 in ceramide-induced autophagic cell death in malignant glioma cells. Cancer Res 64:4286-4293

196. Li X, Fan Z (2010) The epidermal growth factor receptor antibody cetuximab induces autophagy in cancer cells by downregulating HIF-1alpha and Bcl-2 and activating the beclin $1 / \mathrm{hVps} 34$ complex. Cancer Res 70:5942-5952

197. Maubec E, Petrow P, Scheer-Senyarich I, Duvillard P, Lacroix L, Gelly J, Certain A, Duval X, Crickx B, Buffard V et al (2011) Phase II study of cetuximab as first-line single-drug therapy in patients with unresectable squamous cell carcinoma of the skin. J Clin Oncol 29:3419-3426

198. Maycotte P, Aryal S, Cummings CT, Thorburn J, Morgan MJ, Thorburn A (2012) Chloroquine sensitizes breast cancer cells to chemotherapy independent of autophagy. Autophagy $8: 200-212$

199. Duenas-Gonzalez A, Zarba JJ, Patel F, Alcedo JC, Beslija S, Casanova L, Pattaranutaporn P, Hameed S, Blair JM, Barraclough $\mathrm{H}$ et al (2011) Phase III, open-label, randomized study comparing concurrent gemcitabine plus cisplatin and radiation followed by adjuvant gemcitabine and cisplatin versus concurrent cisplatin and radiation in patients with stage IIB to IVA carcinoma of the cervix. J Clin Oncol 29:1678-1685

200. Tsvetkov AS, Miller J, Arrasate M, Wong JS, Pleiss MA, Finkbeiner S (2010) A small-molecule scaffold induces autophagy in primary neurons and protects against toxicity in a Huntington disease model. Proc Natl Acad Sci U S A 107:16982-16987 
201. Nsimba SE (2009) Effects of daily chlorpromazine administration on behavioural and physiological parameters in the rat. Indian $\mathrm{J}$ Physiol Pharmacol 53:209-218

202. Campbell GR, Spector SA (2011) Hormonally active vitamin D3 (1alpha,25-dihydroxycholecalciferol) triggers autophagy in human macrophages that inhibits HIV-1 infection. J Biol Chem 286:18890-18902

203. Vasquez A, Manso G, Cannell J (2004) The clinical importance of vitamin D (cholecalciferol): a paradigm shift with implications for all healthcare providers. Altern Ther Health Med 10:28-36, quiz 37, 94

204. Williams A, Sarkar S, Cuddon P, Ttofi EK, Saiki S, Siddiqi FH, Jahreiss L, Fleming A, Pask D, Goldsmith P et al (2008) Novel targets for Huntington's disease in an mTOR-independent autophagy pathway. Nat Chem Biol 4:295-305

205. Perera PM, Jayamanna SF, Hettiarachchi R, Abeysinghe C, Karunatilake H, Dawson AH, Buckley NA (2009) A phase II clinical trial to assess the safety of clonidine in acute organophosphorus pesticide poisoning. Trials 10:73

206. Zhang T, Li Y, Park KA, Byun HS, Won M, Jeon J, Lee Y, Seok $\mathrm{JH}$, Choi SW, Lee SH et al (2012) Cucurbitacin induces autophagy through mitochondrial ROS production which counteracts to limit caspase-dependent apoptosis. Autophagy 8(4):559-576

207. Ferree A, Guillily M, Li H, Smith K, Takashima A, Squillace R, Weigele M, Collins JJ, Wolozin B (2011) Regulation of physiologic actions of LRRK2: focus on autophagy. Neurodegener Dis 10(1-4):238-241

208. Hartford CM, Desai AA, Janisch L, Karrison T, Rivera VM, Berk L, Loewy JW, Kindler H, Stadler WM, Knowles HL et al (2009) A phase I trial to determine the safety, tolerability, and maximum tolerated dose of deforolimus in patients with advanced malignancies. Clin Cancer Res 15:1428-1434

209. Vara D, Salazar M, Olea-Herrero N, Guzman M, Velasco G, Diaz-Laviada I (2011) Anti-tumoral action of cannabinoids on hepatocellular carcinoma: role of AMPK-dependent activation of autophagy. Cell Death Differ 18:1099-1111

210. Molitoris JK, McColl KS, Swerdlow S, Matsuyama M, Lam M, Finkel TH, Matsuyama S, Distelhorst CW (2011) Glucocorticoid elevation of dexamethasone-induced gene 2 (Dig2/RTP801/ REDD1) protein mediates autophagy in lymphocytes. J Biol Chem 286:30181-30189

211. Bjornson CL, Klassen TP, Williamson J, Brant R, Mitton C, Plint A, Bulloch B, Evered L, Johnson DW (2004) A randomized trial of a single dose of oral dexamethasone for mild croup. N Engl J Med 351:1306-1313

212. Wasko BM, Dudakovic A, Hohl RJ (2011) Bisphosphonates induce autophagy by depleting geranylgeranyl diphosphate. J Pharmacol Exp Ther 337:540-546

213. Diel IJ, Bergner R, Grotz KA (2007) Adverse effects of bisphosphonates: current issues. J Support Oncol 5:475-482

214. Jing K, Song KS, Shin S, Kim N, Jeong S, Oh HR, Park JH, Seo KS, Heo JY, Han J et al (2011) Docosahexaenoic acid induces autophagy through $\mathrm{p} 53 / \mathrm{AMPK} / \mathrm{mTOR}$ signaling and promotes apoptosis in human cancer cells harboring wild-type p53. Autophagy 7:1348-1358

215. Bedikian AY, DeConti RC, Conry R, Agarwala S, Papadopoulos N, Kim KB, Ernstoff M (2011) Phase 3 study of docosahexaenoic acid-paclitaxel versus dacarbazine in patients with metastatic malignant melanoma. Ann Oncol 22:787-793

216. Kobayashi S, Volden P, Timm D, Mao K, Xu X, Liang Q (2010) Transcription factor GATA4 inhibits doxorubicininduced autophagy and cardiomyocyte death. J Biol Chem 285:793-804

217. Kattan J, Droz JP, Couvreur P, Marino JP, Boutan-Laroze A, Rougier P, Brault P, Vranckx H, Grognet JM, Morge X et al (1992) Phase I clinical trial and pharmacokinetic evaluation of doxorubicin carried by polyisohexylcyanoacrylate nanoparticles. Investig New Drugs 10:191-199

218. Shen S, Kepp O, Martins I, Vitale I, Souquere S, Castedo M, Pierron G, Kroemer G (2010) Defective autophagy associated with LC3 puncta in epothilone-resistant cancer cells. Cell Cycle 9:377-383

219. Marino ML, Fais S, Djavaheri-Mergny M, Villa A, Meschini S, Lozupone F, Venturi G, Della Mina P, Pattingre S, Rivoltini L et al (2010) Proton pump inhibition induces autophagy as a survival mechanism following oxidative stress in human melanoma cells. Cell Death Dis 1:e87

220. Travelli C, Drago V, Maldi E, Kaludercic N, Galli U, Boldorini R, Di Lisa F, Tron GC, Canonico PL, Genazzani AA (2011) Reciprocal potentiation of the antitumoral activities of FK866, an inhibitor of nicotinamide phosphoribosyltransferase, and etoposide or cisplatin in neuroblastoma cells. J Pharmacol Exp Ther 338:829-840

221. Sessa C, Zucchetti M, Cerny T, Pagani O, Cavalli F, De Fusco M, De Jong J, Gentili D, McDaniel C, Prins C et al (1995) Phase I clinical and pharmacokinetic study of oral etoposide phosphate. $\mathrm{J}$ Clin Oncol 13:200-209

222. Roscic A, Baldo B, Crochemore C, Marcellin D, Paganetti P (2011) Induction of autophagy with catalytic mTOR inhibitors reduces huntingtin aggregates in a neuronal cell model. J Neurochem 119:398-407

223. Curran MP (2012) Everolimus: in patients with subependymal giant cell astrocytoma associated with tuberous sclerosis complex. Paediatr Drugs 14:51-60

224. Xia HG, Zhang L, Chen G, Zhang T, Liu J, Jin M, Ma X, Ma D, Yuan J (2010) Control of basal autophagy by calpain1 mediated cleavage of ATG5. Autophagy 6:61-66

225. Wurthmann C, Klieser E, Lehmann E (1995) Interaction of therapeutic effects and side effects in drug therapy of generalized anxiety disorders with low dosage fluspirilene. Fortschr Neurol Psychiatr 63:72-77

226. Chouinard G, Annable L, Steinberg S (1986) A controlled clinical trial of fluspirilene, a long-acting injectable neuroleptic, in schizophrenic patients with acute exacerbation. J Clin Psychopharmacol 6:21-26

227. Ravikumar B, Stewart A, Kita H, Kato K, Duden R, Rubinsztein DC (2003) Raised intracellular glucose concentrations reduce aggregation and cell death caused by mutant huntingtin exon 1 by decreasing mTOR phosphorylation and inducing autophagy. Hum Mol Genet 12:985-994

228. Lian J, Karnak D, Xu L (2010) The Bcl-2-Beclin 1 interaction in (-)-gossypol-induced autophagy versus apoptosis in prostate cancer cells. Autophagy 6:1201-1203

229. Gough DR, Cotter TG (2011) Hydrogen peroxide: a Jekyll and Hyde signalling molecule. Cell Death Dis 2:e213

230. Calabretta B, Salomoni P (2012) Suppression of autophagy by BCR/ABL. Front Biosci (Schol Ed) 4:453-460

231. Deininger M, Buchdunger E, Druker BJ (2005) The development of imatinib as a therapeutic agent for chronic myeloid leukemia. Blood 105:2640-2653

232. Christian F, Anthony DF, Vadrevu S, Riddell T, Day JP, McLeod R, Adams DR, Baillie GS, Houslay MD (2010) p62 (SQSTM1) and cyclic AMP phosphodiesterase-4A4 (PDE4A4) locate to a novel, reversible protein aggregate with links to autophagy and proteasome degradation pathways. Cell Signal 22:1576-1596

233. Lim L, Jackson-Lewis V, Wong LC, Shui GH, Goh AX, Kesavapany S, Jenner AM, Fivaz M, Przedborski S, Wenk MR (2012) Lanosterol induces mitochondrial uncoupling and protects dopaminergic neurons from cell death in a model for Parkinson's disease. Cell Death Differ 19:416-427

234. Savaraj N, You M, Wu C, Wangpaichitr M, Kuo MT, Feun LG (2010) Arginine deprivation, autophagy, apoptosis (AAA) for the treatment of melanoma. Curr Mol Med 10:405-412 
235. Haspel J, Shaik RS, Ifedigbo E, Nakahira K, Dolinay T, Englert JA, Choi AM (2011) Characterization of macroautophagic flux in vivo using a leupeptin-based assay. Autophagy 7:629-642

236. Meng N, Zhao J, Su L, Zhao B, Zhang Y, Zhang S, Miao J (2012) A butyrolactone derivative suppressed lipopolysaccharideinduced autophagic injury through inhibiting the autoregulatory loop of p8 and p53 in vascular endothelial cells. Int J Biochem Cell Biol 44:311-319

237. Shimada K, Motoi $Y$, Ishiguro K, Kambe T, Matsumoto SE, Itaya M, Kunichika M, Mori H, Shinohara A, Chiba M et al (2012) Long-term oral lithium treatment attenuates motor disturbance in tauopathy model mice: Implications of autophagy promotion. Neurobiol Dis 46:101-108

238. Mainguet P, Fiasse R (1977) Double-blind placebo-controlled study of loperamide (Imodium) in chronic diarrhoea caused by ileocolic disease or resection. Gut 18:575-579

239. Pan Y, Gao Y, Chen L, Gao G, Dong H, Yang Y, Dong B, Chen X (2011) Targeting autophagy augments in vitro and in vivo antimyeloma activity of DNA-damaging chemotherapy. Clin Cancer Res 17:3248-3258

240. Palumbo A, Bringhen S, Petrucci MT, Musto P, Rossini F, Nunzi M, Lauta VM, Bergonzi C, Barbui A, Caravita T et al (2004) Intermediate-dose melphalan improves survival of myeloma patients aged 50 to 70 : results of a randomized controlled trial. Blood 104:3052-3057

241. Shi WY, Xiao D, Wang L, Dong LH, Yan ZX, Shen ZX, Chen SJ, Chen Y, Zhao WL (2012) Therapeutic metformin/AMPK activation blocked lymphoma cell growth via inhibition of mTOR pathway and induction of autophagy. Cell Death Dis 3:e275

242. Scarpello JH, Howlett HC (2008) Metformin therapy and clinical uses. Diab Vasc Dis Res 5:157-167

243. Olsen EA, Dunlap FE, Funicella T, Koperski JA, Swinehart JM, Tschen EH, Trancik RJ (2002) A randomized clinical trial of $5 \%$ topical minoxidil versus $2 \%$ topical minoxidil and placebo in the treatment of androgenetic alopecia in men. J Am Acad Dermatol 47:377-385

244. Gills JJ, Lopiccolo J, Dennis PA (2008) Nelfinavir, a new anticancer drug with pleiotropic effects and many paths to autophagy. Autophagy 4:107-109

245. Brunner TB, Geiger M, Grabenbauer GG, Lang-Welzenbach M, Mantoni TS, Cavallaro A, Sauer R, Hohenberger W, McKenna WG (2008) Phase I trial of the human immunodeficiency virus protease inhibitor nelfinavir and chemoradiation for locally advanced pancreatic cancer. J Clin Oncol 26:26992706

246. Ofori-Adjei D, Dodoo ANO, Appiah-Danquah A, Couper M (1990) A review of the safety of niclosamide, pyrantel, triclabendazole and oxamniquine. Risk Saf Med 20:113-122

247. Ghanizadeh A, Haghighat R (2012) Nortriptyline for treating enuresis in ADHD-a randomized double-blind controlled clinical trial. Pediatr Nephrol. doi:10.1007/s00467-012-2211-Z

248. Hu C, Zou MJ, Zhao L, Lu N, Sun YJ, Gou SH, Xi T, Guo QL (2012) E Platinum, a newly synthesized platinum compound, induces autophagy via inhibiting phosphorylation of mTOR in gastric carcinoma BGC-823 cells. Toxicol Lett 210:78-86

249. Raymond E, Chaney SG, Taamma A, Cvitkovic E (1998) Oxaliplatin: a review of preclinical and clinical studies. Ann Oncol 9:1053-1071

250. Tong Y, Liu YY, You LS, Qian WB (2012) Perifosine induces protective autophagy and upregulation of ATG5 in human chronic myelogenous leukemia cells in vitro. Acta Pharmacol Sin 33 (4):542-552

251. Vink SR, Schellens JH, Beijnen JH, Sindermann H, Engel J, Dubbelman R, Moppi G, Hillebrand MJ, Bartelink H, Verheij M (2006) Phase I and pharmacokinetic study of combined treatment with perifosine and radiation in patients with advanced solid tumours. Radiother Oncol 80:207-213

252. Cole PL, Beamer AD, McGowan N, Cantillon CO, Benfell K, Kelly RA, Hartley LH, Smith TW, Antman EM (1990) Efficacy and safety of perhexiline maleate in refractory angina. A doubleblind placebo-controlled clinical trial of a novel antianginal agent. Circulation 81:1260-1270

253. Remijsen Q, Vanden Berghe T, Wirawan E, Asselbergh B, Parthoens E, De Rycke R, Noppen S, Delforge M, Willems J, Vandenabeele P (2011) Neutrophil extracellular trap cell death requires both autophagy and superoxide generation. Cell Res 21:290-304

254. Chen D, Fan W, Lu Y, Ding X, Chen S, Zhong Q (2012) A mammalian autophagosome maturation mechanism mediated by TECPR1 and the Atg12-Atg5 conjugate. Mol Cell 45:629-641

255. van Vloten WA (2003) Pimozide: use in dermatology. Dermatol Online J 9:3

256. Chassan JB (1959) A statistical description of a clinical trial of promazine. Psychiatr Q 33:700-714

257. Page CB, Duffull SB, Whyte IM, Isbister GK (2009) Promethazine overdose: clinical effects, predicting delirium and the effect of charcoal. QJM 102:123-131

258. Bahro M, Pfeifer U (1987) Short-term stimulation by propranolol and verapamil of cardiac cellular autophagy. J Mol Cell Cardiol 19:1169-1178

259. Buck ML (2010) Oral propranolol for hemangiomas of infancy. Pediatric Pharmacotherapy 16(8)

260. Liu K, Liu C, Shen L, Shi J, Zhang T, Zhou Y, Zhou L, Sun X (2011) Therapeutic effects of rapamycin on MPTP-induced Parkinsonism in mice. Neurochem Int. doi:10.1016/ j.neuint.2011.05.011

261. Morselli E, Galluzzi L, Kepp O, Criollo A, Maiuri MC, Tavernarakis N, Madeo F, Kroemer G (2009) Autophagy mediates pharmacological lifespan extension by spermidine and resveratrol. Aging (Albany NY) 1:961-970

262. Konrady AO, Kasherininov YR, Shavarov AA, Shavarova EK, Vachrameeva NV, Krutikov AN, Smirnova EV, Shlyakhto EV (2006) How can we block sympathetic overactivity? Effects of rilmenidine and atenolol in overweight hypertensive patients. $\mathrm{J}$ Hum Hypertens 20:398-406. doi:10.1038/sj.jhh.1002004

263. McLean K, VanDeVen NA, Sorenson DR, Daudi S, Liu JR (2009) The HIV protease inhibitor saquinavir induces endoplasmic reticulum stress, autophagy, and apoptosis in ovarian cancer cells. Gynecol Oncol 112:623-630

264. Zorrilla CD, Van Dyke R, Bardeguez A, Acosta EP, Smith B, Hughes MD, Huang S, Watts DH, Heckman B, Jimenez E et al (2007) Clinical response and tolerability to and safety of saquinavir with low-dose ritonavir in human immunodeficiency virus type 1-infected mothers and their infants. Antimicrob Agents Chemother 51:2208-2210

265. Jiang Q, Li F, Shi K, Yang Y, Xu C (2012) Sodium seleniteinduced activation of DAPK promotes autophagy in human leukemia HL60 cells. BMB Rep 45:194-199

266. Bareford MD, Hamed HA, Tang Y, Cruickshanks N, Burow ME, Fisher PB, Moran RG, Nephew KP, Grant S, Dent P (2011) Sorafenib enhances pemetrexed cytotoxicity through an autophagy-dependent mechanism in cancer cells. Autophagy 7:1261-1262

267. Lam ET, Ringel MD, Kloos RT, Prior TW, Knopp MV, Liang J, Sammet S, Hall NC, Wakely PE Jr, Vasko VV et al (2010) Phase II clinical trial of sorafenib in metastatic medullary thyroid cancer. J Clin Oncol 28:2323-2330

268. Tirupathi Pichiah PB, Suriyakalaa U, Kamalakkannan S, Kokilavani P, Kalaiselvi S, SankarGanesh D, Gowri J, Archunan G, Cha YS, Achiraman S (2011) Spermidine may decrease ER stress in pancreatic beta cells and may reduce 
apoptosis via activating AMPK dependent autophagy pathway. Med Hypotheses 77:677-679

269. Jiang J, Maeda A, Ji J, Baty CJ, Watkins SC, Greenberger JS, Kagan VE (2011) Are mitochondrial reactive oxygen species required for autophagy? Biochem Biophys Res Commun 412:55-60

270. Chen Y, Azad MB, Gibson SB (2009) Superoxide is the major reactive oxygen species regulating autophagy. Cell Death Differ 16:1040-1052

271. Bursch W, Ellinger A, Kienzl H, Torok L, Pandey S, Sikorska M, Walker R, Hermann RS (1996) Active cell death induced by the anti-estrogens tamoxifen and ICI 164384 in human mammary carcinoma cells (MCF-7) in culture: the role of autophagy. Carcinogenesis 17:1595-1607

272. Lawrence JA, Adamson PC, Caruso R, Chow C, Kleiner D, Murphy RF, Venzon DJ, Shovlin M, Noone M, Merino M et al (2001) Phase I clinical trial of alitretinoin and tamoxifen in breast cancer patients: toxicity, pharmacokinetic, and biomarker evaluations. J Clin Oncol 19:2754-2763

273. Fujisaka Y, Yamada Y, Yamamoto N, Horiike A, Tamura T (2010) A Phase 1 clinical study of temsirolimus (CCI-779) in Japanese patients with advanced solid tumors. Jpn J Clin Oncol 40:732-738

274. Zeng X, Kinsella TJ (2010) BNIP3 is essential for mediating 6thioguanine- and 5-fluorouracil-induced autophagy following DNA mismatch repair processing. Cell Res 20:665-675

275. Petit E, Langouet S, Akhdar H, Nicolas-Nicolaz C, Guillouzo A, Morel F (2008) Differential toxic effects of azathioprine, 6mercaptopurine and 6-thioguanine on human hepatocytes. Toxicol in Vitro 22:632-642

276. Badham JN, Bardon LM, Reeves PO, Young AM (1963) A trial of thioridazine in mental deficiency. Br J Psychiatry 109:408-410

277. Kruger U, Wang Y, Kumar S, Mandelkow EM (2011) Autophagic degradation of tau in primary neurons and its enhancement by trehalose. Neurobiol Aging 33(10):2291-2305

278. Khunger N, Sarkar R, Jain RK (2004) Tretinoin peels versus glycolic acid peels in the treatment of melasma in dark-skinned patients. Dermatol Surg 30:756-760, discussion 760

279. Takeuchi H, Kondo Y, Fujiwara K, Kanzawa T, Aoki H, Mills GB, Kondo S (2005) Synergistic augmentation of rapamycininduced autophagy in malignant glioma cells by phosphatidylinositol 3-kinase/protein kinase B inhibitors. Cancer Res 65:33363346

280. Klein B, Worndl K, Lutz-Meindl U, Kerschbaum HH (2011) Perturbation of intracellular $\mathrm{K}(+)$ homeostasis with valinomycin promotes cell death by mitochondrial swelling and autophagic processes. Apoptosis 16:1101-1117

281. Duenas-Gonzalez A, Candelaria M, Perez-Plascencia C, PerezCardenas E, de la Cruz-Hernandez E, Herrera LA (2008) Valproic acid as epigenetic cancer drug: preclinical, clinical and transcriptional effects on solid tumors. Cancer Treat Rev 34:206-222

282. Pennock GD, Dalton WS, Roeske WR, Appleton CP, Mosley K, Plezia P, Miller TP, Salmon SE (1991) Systemic toxic effects associated with high-dose verapamil infusion and chemotherapy administration. J Natl Cancer Inst 83:105-110

283. Claerhout S, Lim JY, Choi W, Park YY, Kim K, Kim SB, Lee JS, Mills GB, Cho JY (2011) Gene expression signature analysis identifies vorinostat as a candidate therapy for gastric cancer. PLoS One 6:e24662

284. Ramalingam SS, Kummar S, Sarantopoulos J, Shibata S, LoRusso P, Yerk M, Holleran J, Lin Y, Beumer JH, Harvey RD et al (2010) Phase I study of vorinostat in patients with advanced solid tumors and hepatic dysfunction: a National Cancer Institute Organ Dysfunction Working Group study. J Clin Oncol 28:4507-4512

285. Yang D, Liu H, Goga A, Kim S, Yuneva M, Bishop JM (2010) Therapeutic potential of a synthetic lethal interaction between the
MYC proto-oncogene and inhibition of aurora-B kinase. Proc Natl Acad Sci U S A 107:13836-13841

286. Bauer PO, Wong HK, Oyama F, Goswami A, Okuno M, Kino Y, Miyazaki H, Nukina N (2009) Inhibition of Rho kinases enhances the degradation of mutant huntingtin. J Biol Chem 284:1315313164

287. Chen SY, Chiu LY, Maa MC, Wang JS, Chien CL, Lin WW (2011) zVAD-induced autophagic cell death requires c-Srcdependent ERK and JNK activation and reactive oxygen species generation. Autophagy 7:217-228

288. Ye YC, Yu L, Wang HJ, Tashiro S, Onodera S, Ikejima T (2011) TNFalpha-induced necroptosis and autophagy via supression of the p38-NF-kappa $\beta$ survival pathway in L929 cells. J Pharmacol Sci 117:160-169

289. Major P, Lortholary A, Hon J, Abdi E, Mills G, Menssen HD, Yunus F, Bell R, Body J, Quebe-Fehling E et al (2001) Zoledronic acid is superior to pamidronate in the treatment of hypercalcemia of malignancy: a pooled analysis of two randomized, controlled clinical trials. J Clin Oncol 19:558-567

290. Shen S, Kepp O, Michaud M, Martins I, Minoux H, Metivier D, Maiuri MC, Kroemer RT, Kroemer G (2011) Association and dissociation of autophagy, apoptosis and necrosis by systematic chemical study. Oncogene 30:4544-4556

291. Hill JA, O'Brien JT, Alpert JS, Gore JM, Zusman RM, Christensen D, Boucher CA, Vetrovec G, Borer JS, Friedman C et al (1985) Effect of bepridil in patients with chronic stable angina: results of a multicenter trial. Circulation 71:98-103

292. Lai JH, Ho LJ, Kwan CY, Chang DM, Lee TC (1999) Plant alkaloid tetrandrine and its analog block CD28-costimulated activities of human peripheral blood T cells: potential immunosuppressants in transplantation immunology. Transplantation 68:1383-1392

293. Sharpe DS (1962) A controlled trial of trifluoperazine in the treatment of the mentally subnormal patient. J Ment Sci 108:220-224

294. Lyko F, Brown R (2005) DNA methyltransferase inhibitors and the development of epigenetic cancer therapies. J Natl Cancer Inst 97:1498-1506

295. Kaminskas E, Farrell A, Abraham S, Baird A, Hsieh LS, Lee SL, Leighton JK, Patel H, Rahman A, Sridhara R et al (2005) Approval summary: azacitidine for treatment of myelodysplastic syndrome subtypes. Clin Cancer Res 11:3604-3608

296. Cashen AF, Schiller GJ, O'Donnell MR, DiPersio JF (2010) Multicenter, phase II study of decitabine for the first-line treatment of older patients with acute myeloid leukemia. J Clin Oncol 28:556-561

297. Merskey H (1958) A clinical and psychometric study of the effects of procaine amide in Huntington's chorea. J Ment Sci 104:411-420

298. Mereles D, Hunstein W (2011) Epigallocatechin-3-gallate (EGCG) for clinical trials: more pitfalls than promises? Int $\mathrm{J}$ Mol Sci 12:5592-5603

299. Singh N, Duenas-Gonzalez A, Lyko F, Medina-Franco JL (2009) Molecular modeling and molecular dynamics studies of hydralazine with human DNA methyltransferase 1. ChemMedChem 4:792-799

300. Stiell IG, Clement CM, Symington C, Perry JJ, Vaillancourt C, Wells GA (2007) Emergency department use of intravenous procainamide for patients with acute atrial fibrillation or flutter. Acad Emerg Med 14:1158-1164

301. Liu Z, Liu S, Xie Z, Pavlovicz RE, Wu J, Chen P, Aimiuwu J, Pang J, Bhasin D, Neviani P et al (2009) Modulation of DNA methylation by a sesquiterpene lactone parthenolide. J Pharmacol Exp Ther 329:505-514

302. Curry EA 3rd, Murry DJ, Yoder C, Fife K, Armstrong V, Nakshatri H, O'Connell M, Sweeney CJ (2004) Phase I dose escalation trial of feverfew with standardized doses of parthenolide in patients with cancer. Investig New Drugs 22:299-305 
303. Liu Z, Xie Z, Jones W, Pavlovicz RE, Liu S, Yu J, Li PK, Lin J, Fuchs JR, Marcucci G et al (2009) Curcumin is a potent DNA hypomethylation agent. Bioorg Med Chem Lett 19:706-709

304. Hsu CH, Cheng AL (2007) Clinical studies with curcumin. Adv Exp Med Biol 595:471-480

305. Lin RK, Hsu CH, Wang YC (2007) Mithramycin A inhibits DNA methyltransferase and metastasis potential of lung cancer cells. Anticancer Drugs 18:1157-1164

306. Liacini A, Sylvester J, Li WQ, Zafarullah M (2005) Mithramycin downregulates proinflammatory cytokine-induced matrix metalloproteinase gene expression in articular chondrocytes. Arthritis Res Ther 7:R777-R783

307. Kuck D, Singh N, Lyko F, Medina-Franco JL (2010) Novel and selective DNA methyltransferase inhibitors: docking-based virtual screening and experimental evaluation. Bioorg Med Chem $18: 822-829$

308. Kuck D, Caulfield T, Lyko F, Medina-Franco JL (2010) Nanaomycin A selectively inhibits DNMT3B and reactivates silenced tumor suppressor genes in human cancer cells. Mol Cancer Ther 9:3015-3023 